Research Article

\title{
Some Existence Results for a System of Nonlinear Fractional Differential Equations
}

\author{
Eskandar Ameer, ${ }^{1}$ Hassen Aydi $\left(\mathbb{D},{ }^{2,3}\right.$ Hüseyin Işık (iD), ${ }^{4}$ Muhammad Nazam (iD, ${ }^{5}$ \\ Vahid Parvaneh $\left(\mathbb{D},{ }^{6}\right.$ and Muhammad Arshad $\mathbb{B D}^{7}$ \\ ${ }^{1}$ Department of Mathematics, Taiz University, Taiz, Yemen \\ ${ }^{2}$ Université de Sousse, Institut Supérieur d'Informatique et des Techniques de Communication, H. Sousse 4000, Tunisia \\ ${ }^{3}$ China Medical University Hospital, China Medical University, Taichung 40402, Taiwan \\ ${ }^{4}$ Department of Mathematics, Muş Alparslan University, Muş 49250, Turkey \\ ${ }^{5}$ Department of Mathematics, Allama Iqbal Open University, H-8, Islamabad, Pakistan \\ ${ }^{6}$ Department of Mathematics, Gilan-E-Gharb Branch, Islamic Azad University, Gilan-E-Gharb, Iran \\ ${ }^{7}$ Department of Mathematics and Statistics, International Islamic University, Islamabad, Pakistan
}

Correspondence should be addressed to Hassen Aydi; hassen.aydi@isima.rnu.tn and Vahid Parvaneh; zam.dalahoo@gmail.com

Received 16 June 2020; Revised 2 October 2020; Accepted 22 October 2020; Published 19 November 2020

Academic Editor: Serkan Araci

Copyright ( $) 2020$ Eskandar Ameer et al. This is an open access article distributed under the Creative Commons Attribution License, which permits unrestricted use, distribution, and reproduction in any medium, provided the original work is properly cited.

In this paper, we show that a sequence satisfying a Suzuki-type JS-rational contraction or a generalized Suzuki-type Ćirić JScontraction, under some conditions, is a Cauchy sequence. This paper presents some common fixed point theorems and an application to resolve a system of nonlinear fractional differential equations. Some examples and consequences are also given.

\section{Introduction}

The application of Banach contraction principle (BCP) [1] is wide spread. Recently, fixed point theory is being applied to show the existence of solutions of different mathematical models expressed in the forms of differential, integral, functional, fractional differential, and matrix equations (both linear and nonlinear). There are several common fixed point theorems, in the literature, which generalize BCP and have been applied to show the existence of solutions of different mathematical models involving two or more functions (see [2-19], for details).

Suzuki [20] presented a new generalization of BCP, so called a Suzuki-contraction, and established an existence theorem which characterized the metric completeness. Piri and Kumam [21, 22] investigated results in [20] under the $F$-contraction structure both in metric and $b$-metric spaces, respectively. Further generalizations of Suzuki contractions have been studied by Aydi et al. [23] who introduced a Suzuki-type multivalued contraction to obtain fixed point theorems in the setting of weak partial metric spaces. Abbas et al. [2] discussed generalized Suzuki-type multivalued contractions under the effect of a binary relation and obtained some fixed point results.

On the contrary, Jleli et al. [24, 25] introduced another generalization of BCP, known as a JS-contraction (also known as a $\theta$-contraction) and $\mathrm{Li}$ and Jiang [26] modified the JS-contraction to JS-quasi contractions in order to obtain more general fixed point results, as compared to [24]. Altun et al. [27] obtained some fixed point theorems for JS-contraction type mappings via a binary operation. Several honorable researchers have published their valuable investigations on JS-contractions in well ranked journals (see [28-31]).

In this paper, we structure two common fixed point theorems comprising four self-mappings involving generalized Suzuki-type $\theta$-rational contractions and generalized 
Suzuki-type Ćirić $\theta$-contractions. The existence of the solution of a mathematical model in terms of fractional differential equations is shown through a common fixed point theorem.

\section{Advances on $\theta$-Contractions}

Let $\Theta:=\left\{\theta:(0, \infty) \longrightarrow(1, \infty)\right.$ such that $\theta$ satisfies $\Theta_{1}, \Theta_{2}$, $\left.\Theta_{3}\right\}$, where

$\left(\Theta_{1}\right) \theta$ is nondecreasing.

$\left(\Theta_{2}\right)$ For each sequence $\left\{\breve{t}_{n}\right\} \subset(0, \infty)$,

$\lim _{n \longrightarrow \infty} \theta\left(\breve{t}_{n}\right)=1, \quad$ if and only if $\lim _{n \longrightarrow \infty} \breve{t}_{n}=0$.

$\left(\Theta_{3}\right)$ There exist $q \in(0,1)$ and $\ell \in(0, \infty]$ such that $\lim _{\breve{t} \longrightarrow 0^{+}} \theta(\breve{t})-1 / \breve{t}^{q}=\ell$.

Jleli and Samet [24] introduced the following.

Definition 1. Let $(\chi, \rho)$ be a metric space. The mapping $T: \chi \longrightarrow \chi$ is called a $\theta$-contraction (or a JS-contraction) if there exist a constant $k \in[0,1)$ and $\theta \in \Theta$ such that

$$
r, j \in \chi, \quad \rho(\breve{T r}, \breve{T} j) \neq 0 \Rightarrow \theta(\rho(\breve{T r}, \breve{T} j)) \leq[\theta(\rho(r, j))]^{k} .
$$

The following examples show that the set $\Theta$ is not empty.

Example 1. Let $\Phi, \psi:(0, \infty) \longrightarrow(1, \infty)$ be defined by, for all $t \in(0, \infty)$,

$$
\begin{aligned}
& \Phi(t)=e^{\sqrt{t}}, \\
& \psi(t)=e^{\sqrt{t e^{t}}} .
\end{aligned}
$$

Then, $\Phi$ and $\psi$ are in $\Theta$. theorem.

Jleli and Samet [24] established the following fixed point

Theorem 1 (see [24]). Let $(\chi, \rho)$ be a complete metric space and $T: \chi \longrightarrow \chi$ be a $\theta$-contraction. Then, $T$ has a unique fixed point.

Consistent with [28], let $\mu:=\{\theta:(0, \infty) \longrightarrow$ $(1, \infty) \mid \theta$ satisfy $\left.\left(\Theta_{1}\right),\left(\Theta_{2}\right),\left(\Theta_{3}^{\prime}\right)\right\}$, where

$\left(\Theta^{\prime} 3\right) \theta$ is continuous on $(0, \infty)$.

Note that $(\Theta 3)$ and $\left(\Theta^{\prime} 3\right)$ are independent of each other. For this, see examples in [28]. The set of functions $\mu$ is not empty, as shown in the following.

Example 2 (see [28]). Let $\phi, \varphi, \psi, F, G, H:(0, \infty) \longrightarrow$ $(1, \infty)$ be defined by, for all $t \in(0, \infty)$,

$$
\begin{aligned}
& \phi(t)=e^{t}, \\
& \varphi(t)=\cos h t, \\
& \psi(t)=e^{\sqrt{t e^{t}}}, \\
& F(t)=1+\ln (t+1), \\
& G(t)=e^{\sqrt{t}}, \\
& H(t)=e^{t e^{t}} .
\end{aligned}
$$

Here, $\phi, \varphi, \psi, F, G, H \in \mu$.

Hussain et al. [29] modified the class $\Theta$ of mappings as follows:

Let $\Psi=\left\{\theta:[0, \infty) \longrightarrow[1, \infty): \theta\right.$ satisfies $\left.\left(\Psi_{1}\right)-\left(\Psi_{5}\right)\right\}$,

where

$\left(\Psi_{1}\right) \theta$ is non decreasing.

$\left(\Psi_{2}\right) \theta(\breve{t})=1$ if and only if $\breve{t}=0$.

$\left(\Psi_{3}\right)$ For each sequence $\left\{\breve{t}_{n}\right\} \subset(0, \infty)$ :

$\lim _{n \longrightarrow \infty} \theta\left(\breve{t}_{n}\right)=1, \quad$ if and only if $\lim _{n \longrightarrow \infty} \breve{t}_{n}=0$.

$\left(\Psi_{4}\right)$ There exist $g_{q} \in(0,1)$ and $\ell \in(0, \infty]$ such that $\lim _{\dot{t} \longrightarrow 0^{+}} \theta(\breve{t})-1 / t^{q}=\ell$.

$\left(\Psi_{5}\right) \theta\left(\breve{t}_{1}+\breve{t}_{2}\right) \leq \theta\left(\breve{t}_{1}\right) \theta\left(\breve{t}_{2}\right)$.

The functions $f(t)=e^{\sqrt{t}}$ and $g(t)=5^{\sqrt{t}}$ (for all $t \in[0, \infty))$ are in $\Psi$. $\left(E^{*}\right)$ : for all $t_{1}, t_{2}, t_{3}, t_{4} \in \mathbb{R}^{+}$with $t_{1} \cdot t_{2} \cdot t_{3} \cdot t_{4}=0$, there exists $k \in[0,1)$ such that $E\left(t_{1}, t_{2}, t_{3}, t_{4}\right)=k$.

Let $\Delta_{E}$ denote the set of all functions $E: \mathbb{R}^{+4} \longrightarrow \mathbb{R}^{+}$ satisfying the condition $\left(E^{*}\right)$.

Example 3 (see [31]). If $E\left(t_{1}, t_{2}, t_{3}, t_{4}\right)=\lambda e^{v \min \left\{t_{1}, t_{2}, t_{3}, t_{4}\right\}}$, where $v \in \mathbb{R}^{+}$and $\lambda \in[0,1)$, then $E \in \Delta_{E}$.

Example 4 (see [31]). If $E\left(t_{1}, t_{2}, t_{3}, t_{4}\right)=m \cdot \min$ $\left\{t_{1}, t_{2}, t_{3}, t_{4}\right\}+\lambda$, where $m \in \mathbb{R}^{+}$and $\lambda \in[0,1)$, then $E \in \Delta_{E}$.

The following class of mappings was considered in [32] to develop some fixed point theorems. We also use this class of mappings in our investigations:

$$
\Phi=\left\{\Upsilon: \mathbb{R}^{+} \times \mathbb{R}^{+} \longrightarrow \mathbb{R} \text { and } \Upsilon(s, t)=\frac{1}{2} s-t\right\} .
$$

Definition 2 (see [5]). Let $(\chi, \rho)$ be a metric space. The pair $\{f, g\}$ is said to be compatible if and only if

$$
\lim _{n \longrightarrow \infty} \rho\left(f\left(g\left(r_{n}\right)\right), g\left(f\left(r_{n}\right)\right)\right)=0,
$$

whenever $\left\{r_{n}\right\}$ is a sequence in $\chi$ so that

$$
\lim _{n \longrightarrow \infty} f\left(r_{n}\right)=\lim _{n \longrightarrow \infty} g\left(r_{n}\right)=t, \quad \text { for some } t \in \chi .
$$


The following lemma is important in the sequel.

Lemma 1 (see [5]). Let $(\chi, \rho)$ be a metric space. If there exist two sequences $\left\{r_{n}\right\}$ and $\left\{u_{n}\right\}$ such that

$$
\begin{aligned}
\lim _{n \longrightarrow \infty} \rho\left(r_{n}, u_{n}\right) & =0, \\
& \lim _{n \longrightarrow \infty} r_{n}=t, \quad \text { for some } t \in \chi,
\end{aligned}
$$

then

$$
\lim _{n \longrightarrow \infty} u_{n}=t
$$

\section{Generalized Suzuki-Type Contractions and Fixed Point Results}

Jleli and Samet $[24,25]$ have employed $\theta$-contractions to obtain some fixed point theorems. Suzuki [20] extended Edelstein contraction to develop a new generalization of Banach contraction called Suzuki contraction. The contractions developed in $[20,24]$ were then followed by Liu et al. [33] to introduce a Suzuki-type $\theta$-contraction. In this section, we introduce more general forms of Suzuki-type $\theta$-contractions involving four self-mappings. We construct some conditions under which a sequence, whose terms satisfy generalized Suzuki-type $\theta$-rational contractions or generalized Suzuki-type Ćirić $\theta$-contractions, is a Cauchy sequence. We obtain two common fixed point theorems, which then are applied to show the existence of the solution of a system of fractional differential equations. We start with the following definition.

Definition 3. Let $(\chi, \rho)$ be a metric space, and $f, g, \widehat{S}, T: \chi \longrightarrow \chi$ be four single-valued maps. These maps form a new generalized Suzuki-type $(\theta, E)$-rational contraction if, for all $r, j \in \chi$, with $\rho(f r, g j)>0$, for some $\theta \in \mu$, $E \in \Delta_{E}$, and $\Upsilon \in \Phi$,

$$
\Upsilon(\rho(f r, \widehat{S} r), \rho(\widehat{S} r, \breve{T} j))<0 \Rightarrow \theta(\rho(f r, g j)) \leq[\theta(A(r, j))]^{E(G(r, j))},
$$

where

$$
\begin{aligned}
& A(r, j)=\max \left\{\begin{array}{c}
\rho(\widehat{S} r, \breve{T} j), \rho(f r, \widehat{S} r), \rho(g j, \breve{T} j), \frac{\rho(f r, \breve{T} j)+\rho(g j, \widehat{S} r)}{2}, \\
\frac{(1+\rho(f r, \widehat{S} r)) \rho(g j, \breve{T} j)}{1+\rho(\widehat{S} r, \breve{T} j)}
\end{array}\right\}, \\
& G(r, j)=\{\rho(f r, \widehat{S} r), \rho(g j, \breve{T} j), \rho(f r, \breve{T} j), \rho(g j, \widehat{S} r)\} .
\end{aligned}
$$

Our first result is as follows.

Theorem 2. Let $(\chi, \rho)$ be a complete metric space, and $f, g, \widehat{S}, T: \chi \longrightarrow \chi$ be four single-valued maps. Suppose that the mappings $f, g, \widehat{S}, T$ form a new generalized Suzuki-type $(\theta, E)$-rational contraction with $f(\chi) \subseteq T(\chi)$ and $g(\chi) \subseteq \widehat{S}(\chi)$. If $(f, \widehat{S})$ and $(g, \widehat{T})$ are compatible pair and $T$ and $\widehat{S}$ are continuous on $(\chi, \rho)$, then $f, g, \widehat{S}$, and $\widehat{T}$ have a unique common fixed point in $\chi$.

Proof. Let $r_{0} \in \chi$ be an arbitrary point. As $f(\chi) \subseteq \breve{T}(\chi)$, there exists $r_{0} \in \chi$ such that

$$
f\left(r_{0}\right)=\breve{T}\left(r_{1}\right)
$$

Since $g\left(r_{1}\right) \in \widehat{S}(\chi)$, we can choose $r_{2} \in \chi$ such that $g\left(r_{1}\right)=\widehat{S}\left(r_{2}\right)$. In general $r_{2 n+1}$ and $r_{2 n+2}$ are chosen in $\chi$ such that

$$
\begin{aligned}
f\left(r_{2 n}\right) & =\breve{T}\left(r_{2 n+1}\right), \\
g\left(r_{2 n+1}\right) & =\widehat{S}\left(r_{2 n+2}\right) .
\end{aligned}
$$

We construct a sequence $\left\{\Re_{n}\right\}$ in $\chi$ such that

$$
\begin{gathered}
\mathfrak{R}_{2 n}=f\left(r_{2 n}\right)=\breve{T}\left(r_{2 n+1}\right), \\
\mathfrak{R}_{2 n+1}=g\left(r_{2 n+1}\right)=\widehat{S}\left(r_{2 n+2}\right),
\end{gathered}
$$

for $n=0,1,2, \ldots$. Assume that $\rho\left(f\left(r_{2 n}\right), g\left(r_{2 n+1}\right)\right)>0$ and since

$$
\begin{aligned}
\frac{1}{2} \rho\left(f\left(r_{2 n}\right), \widehat{S}\left(r_{2 n}\right)\right) & =\frac{1}{2} \rho\left(\mathfrak{R}_{2 n}, \mathfrak{R}_{2 n-1}\right)<\rho\left(\mathfrak{R}_{2 n-1}, \mathfrak{R}_{2 n}\right) \\
& =\rho\left(\widehat{S}\left(r_{2 n}\right), \breve{T}\left(r_{2 n+1}\right)\right) .
\end{aligned}
$$

We have

$$
\Upsilon\left(\rho\left(f\left(r_{2 n}\right), \widehat{S}\left(r_{2 n}\right)\right), \rho\left(\widehat{S}\left(r_{2 n}\right), \breve{T}\left(r_{2 n+1}\right)\right)\right)<0 .
$$

Hence, from the contractive condition (12),

$$
\begin{aligned}
1<\theta\left(\rho\left(\mathfrak{R}_{2 n}, \mathfrak{R}_{2 n+1}\right)\right) & =\theta\left(\rho\left(f\left(r_{2 n}\right), g\left(r_{2 n+1}\right)\right)\right) \\
& \leq\left[\theta\left(A\left(r_{2 n}, r_{2 n+1}\right)\right)\right]^{E\left(G\left(r_{2 n}, r_{2 n+1}\right)\right)} .
\end{aligned}
$$

For every $n \geq 1$, where 


$$
\begin{aligned}
G\left(r_{2 n}, r_{2 n+1}\right) & =\left\{\begin{array}{c}
\rho\left(\mathfrak{R}_{2 n}, \mathfrak{R}_{2 n-1}\right), \rho\left(\mathfrak{R}_{2 n+1}, \mathfrak{R}_{2 n}\right), \\
\rho\left(\mathfrak{R}_{2 n}, \mathfrak{R}_{2 n}\right), \rho\left(\mathfrak{R}_{2 n+1}, \mathfrak{R}_{2 n-1}\right)
\end{array}\right\} \\
& =\left\{\begin{array}{c}
\rho\left(\mathfrak{R}_{2 n}, \mathfrak{R}_{2 n-1}\right), \rho\left(\mathfrak{R}_{2 n+1}, \mathfrak{R}_{2 n}\right), \\
0, \rho\left(\mathfrak{R}_{2 n+1}, \mathfrak{R}_{2 n-1}\right)
\end{array}\right\} .
\end{aligned}
$$

$$
\begin{aligned}
E\left(G\left(r_{2 n}, r_{2 n+1}\right)\right)= & E\left(\rho\left(\mathfrak{R}_{2 n}, \mathfrak{R}_{2 n-1}\right), \rho\left(\mathfrak{R}_{2 n+1}, \mathfrak{R}_{2 n}\right), 0, \rho\right. \\
& \left.\cdot\left(\mathfrak{R}_{2 n+1}, \mathfrak{R}_{2 n-1}\right)\right)=z,
\end{aligned}
$$

As $\rho\left(\mathfrak{R}_{2 n}, \mathfrak{R}_{2 n-1}\right) \cdot \rho\left(\mathfrak{R}_{2 n+1}, \mathfrak{R}_{2 n}\right) \cdot \rho\left(\mathfrak{R}_{2 n+1}, \mathfrak{R}_{2 n-1}\right) \cdot 0=0$, so by $\left(E^{*}\right)$, there exists $z \in[0,1)$ such that

$$
\begin{aligned}
& A\left(r_{2 n}, r_{2 n+1}\right)=\max \left\{\begin{array}{c}
\rho\left(\widehat{S} r_{2 n}, \breve{T} r_{2 n+1}\right), \rho\left(f r_{2 n}, \widehat{S} r_{2 n}\right), \rho\left(g r_{2 n+1}, \breve{T} r_{2 n+1}\right), \\
\frac{\rho\left(f r_{2 n}, \breve{T} r_{2 n+1}\right)+\rho\left(g r_{2 n+1}, \widehat{S} r_{2 n}\right)}{2}, \frac{\left(1+\rho\left(f r_{2 n}, \widehat{S} r_{2 n}\right)\right) \rho\left(g r_{2 n+1}, \breve{T} r_{2 n+1}\right)}{1+\rho\left(\widehat{S} r_{2 n}, \breve{T} r_{2 n+1}\right)},
\end{array}\right\} \\
& =\max \left\{\begin{array}{c}
\rho\left(\mathfrak{R}_{2 n-1}, \mathfrak{R}_{2 n}\right), \rho\left(\mathfrak{R}_{2 n}, \mathfrak{R}_{2 n-1}\right), \rho\left(\mathfrak{R}_{2 n+1}, \mathfrak{R}_{2 n}\right), \\
\frac{\rho\left(\boldsymbol{R}_{2 n}, \mathfrak{R}_{2 n}\right)+\rho\left(\mathfrak{R}_{2 n+1}, \mathfrak{R}_{2 n-1}\right)}{2}, \frac{\left(1+\rho\left(\boldsymbol{R}_{2 n}, \mathfrak{R}_{2 n-1}\right)\right) \rho\left(\boldsymbol{R}_{2 n+1}, \mathfrak{R}_{2 n}\right)}{1+\rho\left(\mathfrak{R}_{2 n-1}, \mathfrak{R}_{2 n}\right)},
\end{array}\right\} \\
& =\max \left\{\rho\left(\mathfrak{R}_{2 n-1}, \mathfrak{R}_{2 n}\right), \rho\left(\mathfrak{R}_{2 n+1}, \mathfrak{R}_{2 n}\right), \frac{\rho\left(\mathfrak{R}_{2 n+1}, \mathfrak{R}_{2 n-1}\right)}{2}\right\} .
\end{aligned}
$$

Since

$$
\begin{aligned}
\frac{\rho\left(\mathfrak{R}_{2 n+1}, \mathfrak{R}_{2 n-1}\right)}{2} & \leq \frac{\rho\left(\mathfrak{R}_{2 n}, \mathfrak{R}_{2 n-1}\right)}{2}+\frac{\rho\left(\mathfrak{R}_{2 n+1}, \mathfrak{R}_{2 n}\right)}{2} \\
& \leq \max \left\{\rho\left(\mathfrak{R}_{2 n-1}, \mathfrak{R}_{2 n}\right), \rho\left(\mathfrak{R}_{2 n+1}, \mathfrak{R}_{2 n}\right)\right\} .
\end{aligned}
$$

one writes

$$
A\left(r_{2 n}, r_{2 n+1}\right)=\max \left\{\rho\left(\mathfrak{R}_{2 n-1}, \mathfrak{R}_{2 n}\right), \rho\left(\mathfrak{R}_{2 n+1}, \mathfrak{R}_{2 n}\right)\right\} .
$$

If, for some $n, A\left(r_{2 n}, r_{2 n+1}\right)=\rho\left(\mathfrak{R}_{2 n}, \mathfrak{R}_{2 n+1}\right)$, then from (19), (21), and (24), we have

$$
\theta\left(\rho\left(\mathfrak{R}_{2 n}, \mathfrak{R}_{2 n+1}\right)\right) \leq\left[\theta\left(\rho\left(\mathfrak{R}_{2 n}, \mathfrak{R}_{2 n+1}\right)\right)\right]^{z}<\theta\left(\rho\left(\mathfrak{R}_{2 n}, \mathfrak{R}_{2 n+1}\right)\right),
$$

a contradiction. Therefore,

$\theta\left(\rho\left(\mathfrak{R}_{2 n}, \mathfrak{R}_{2 n+1}\right)\right) \leq\left[\theta\left(\rho\left(\mathfrak{R}_{2 n-1}, \mathfrak{R}_{2 n}\right)\right)\right]^{z}, \quad$ for $n \geq 1$.

Thus, for all $n \in \mathbb{N}$, we have

$\theta\left(\rho\left(\mathfrak{R}_{n}, \mathfrak{R}_{n+1}\right)\right) \leq\left[\theta\left(\rho\left(\mathfrak{R}_{n-1}, \mathfrak{R}_{n}\right)\right)\right]^{z}, \quad$ for $n \geq 1$.

It implies that

$$
\begin{aligned}
1 & <\theta\left(\rho\left(\mathfrak{R}_{n}, \mathfrak{R}_{n+1}\right)\right) \\
& \leq\left[\theta\left(\rho\left(\mathfrak{R}_{n-1}, \mathfrak{R}_{n}\right)\right)\right]^{z^{2}} \\
& \vdots \\
& \leq\left[\theta\left(\rho\left(\mathfrak{R}_{0}, \mathfrak{R}_{1}\right)\right)\right]^{z^{n}},
\end{aligned}
$$

for all $n \in \mathbb{N}$. Taking the limit as $n \longrightarrow \infty$ in (28) and using the fact that $\theta \in \mu$, we have

$$
\lim _{n \longrightarrow \infty} \theta\left(\rho\left(\mathfrak{R}_{n}, \mathfrak{R}_{n+1}\right)\right)=1 .
$$

By $(\Theta 2)$, we obtain

$$
\lim _{n \rightarrow \infty} \rho\left(\Re_{n}, \Re_{n+1}\right)=0 .
$$

Now, we will show that $\left\{\mathfrak{R}_{n}\right\}$ is a Cauchy sequence. We suppose on the contrary that $\left\{\mathfrak{R}_{n}\right\}$ is not a Cauchy sequence; then, there exist $\varepsilon>0$ and subsequences $\{n(k)\}_{k=1}^{\infty}$ and $\{m(k)\}_{k=1}^{\infty}$ of natural numbers such that, for $m(k)>n(k)>k$, we have $\rho\left(\mathfrak{R}_{n(k)}, \mathfrak{R}_{m(k)}\right) \geq \varepsilon$. Then, $\rho\left(\mathfrak{R}_{n(k)}, \mathfrak{R}_{m(k)-1}\right)<\varepsilon$ for all $k \in \mathbb{N}$. Therefore,

$$
\begin{aligned}
\varepsilon & \leq \rho\left(\mathfrak{R}_{n(k)}, \mathfrak{R}_{m(k)}\right) \leq \rho\left(\mathfrak{R}_{n(k)}, \mathfrak{R}_{m(k)-1}\right)+\rho\left(\mathfrak{R}_{m(k)-1}, \mathfrak{R}_{m(k)}\right) \\
& <\varepsilon+\rho\left(\boldsymbol{R}_{m(k)-1}, \mathfrak{R}_{m(k)}\right) .
\end{aligned}
$$

By taking the upper limit as $k \longrightarrow \infty$ in (31) and using (30), we obtain 


$$
\lim _{k \longrightarrow \infty} \rho\left(\mathfrak{R}_{n(k)}, \Re_{m(k)}\right)=\varepsilon .
$$

Using the triangular inequality, we have

$$
\rho\left(\mathfrak{R}_{n(k)}, \mathfrak{R}_{m(k)}\right) \leq \rho\left(\mathfrak{R}_{n(k)}, \mathfrak{R}_{n(k)+1}\right)+\rho\left(\mathfrak{R}_{n(k)+1}, \mathfrak{R}_{m(k)}\right),
$$

$$
\rho\left(\Re_{n(k)+1}, \mathfrak{R}_{m(k)}\right) \leq \rho\left(\Re_{n(k)+1}, \mathfrak{R}_{n(k)}\right)+\rho\left(\Re_{n(k)}, \mathfrak{R}_{m(k)}\right) .
$$

By taking upper limit as $k \longrightarrow \infty$ in (33) and (34) and applying (30) and (32),

$$
\varepsilon \leq \lim _{k \longrightarrow \infty} \rho\left(\Re_{n(k)+1}, \Re_{m(k)}\right) \leq \varepsilon .
$$

Thus,

$$
\lim _{k \longrightarrow \infty} \rho\left(\Re_{n(k)+1}, \mathfrak{R}_{m(k)}\right)=\varepsilon .
$$

Similarly, we can obtain

$\lim _{k \longrightarrow \infty} \rho\left(\Re_{n(k)+1}, \Re_{m(k)+1}\right)=\lim _{k \longrightarrow \infty} \rho\left(\Re_{n(k)}, \mathfrak{R}_{m(k)+1}\right)=\varepsilon$.

Assume that $\rho\left(f\left(r_{n(k)+1}\right), g\left(r_{m(k)+1}\right)\right)>0$, and since

$$
\frac{1}{2} \rho\left(f\left(r_{n(k)}\right), \widehat{S}\left(r_{m(k)}\right)\right)=\frac{1}{2} \rho\left(\mathfrak{R}_{n(k)}, \mathfrak{R}_{m(k)-1}\right)
$$

$$
<\rho\left(\mathfrak{R}_{n(k)}, \mathfrak{R}_{m(k)-1}\right)
$$$$
=\rho\left(\widehat{S}\left(r_{m(k)}\right), \breve{T}\left(r_{n(k)+1}\right)\right) \text {, }
$$

we obatin

$$
\Upsilon\left(\rho\left(f\left(r_{n(k)}\right), \widehat{S}\left(r_{m(k)}\right)\right), \rho\left(\widehat{S}\left(r_{n(k)}\right), \breve{T}\left(r_{m(k)+1}\right)\right)\right)<0 .
$$

Hence, from (12),

$$
\begin{aligned}
\theta\left(\rho\left(\mathfrak{R}_{n(k)+1}, \mathfrak{R}_{m(k)+1}\right)\right) & =\theta\left(\rho\left(f\left(r_{n(k)+1}\right), g\left(r_{m(k)+1}\right)\right)\right) \\
& \leq\left[\theta\left(A\left(r_{n(k)}, r_{m(k)}\right)\right)\right]^{E\left(G\left(r_{n(k)}, r_{m(k)}\right)\right)},
\end{aligned}
$$

where

$$
G\left(r_{n(k)}, r_{m(k)}\right)=\left\{\begin{array}{c}
\rho\left(\Re_{n(k)+1}, \mathfrak{R}_{n(k)}\right), \rho\left(\mathfrak{R}_{m(k)+1}, \mathfrak{R}_{m(k)}\right), \\
\rho\left(\mathfrak{R}_{n(k)+1}, \mathfrak{R}_{m(k)}\right), \rho\left(\mathfrak{R}_{m(k)+1}, \mathfrak{R}_{n(k)}\right)
\end{array}\right\} .
$$

Taking limit at $k \longrightarrow \infty$ in (41), we obtain

$$
\lim _{k \longrightarrow \infty} G\left(r_{n(k)}, r_{m(k)}\right)=\left\{\begin{array}{c}
\lim _{k \longrightarrow \infty} \rho\left(\mathfrak{R}_{n(k)+1}, \mathfrak{R}_{n(k)}\right), \lim _{k \longrightarrow \infty} \rho\left(\mathfrak{R}_{m(k)+1}, \mathfrak{R}_{m(k)}\right), \\
\lim _{k \longrightarrow \infty} \rho\left(\mathfrak{R}_{n(k)+1}, \mathfrak{R}_{m(k)}\right), \lim _{k \longrightarrow \infty} \rho\left(\mathfrak{R}_{m(k)+1}, \mathfrak{R}_{n(k)}\right)
\end{array}\right\}=\{0,0, \mathcal{\varepsilon}, \varepsilon\}
$$

By $E^{*}$, there exists $z \in[0,1)$ such that $E(0,0, \varepsilon, \varepsilon)=z$. Using the continuity of $E$ and (41),

$$
E\left(\lim _{k \rightarrow \infty} G\left(r_{n(k)}, r_{m(k)}\right)\right) \leq z .
$$

Also,

$$
A\left(r_{n(k)}, r_{m(k)}\right)=\max \left\{\begin{array}{c}
\rho\left(\widehat{S}\left(r_{n(k)+1}\right), \breve{T}\left(r_{m(k)+1}\right)\right), \rho\left(f\left(r_{n(k)+1}\right), \widehat{S}\left(r_{n(k)+1}\right)\right), \\
\rho\left(g\left(r_{m(k)+1}\right), \breve{T}\left(r_{m(k)+1}\right)\right), \\
\frac{\rho\left(f\left(r_{n(k)+1}\right), \breve{T}\left(r_{m(k)+1}\right)\right)+\rho\left(g\left(r_{m(k)+1}\right), \widehat{S}\left(r_{n(k)+1}\right)\right)}{2}, \\
\frac{\left(1+\rho\left(f\left(r_{n(k)+1}\right), \widehat{S}\left(r_{n(k)+1}\right)\right)\right) \rho\left(g\left(r_{m(k)+1}\right), \breve{T}\left(r_{m(k)+1}\right)\right)}{1+\rho\left(\widehat{S}\left(r_{n(k)+1}\right), \breve{T}\left(r_{m(k)+1}\right)\right)}
\end{array}\right\},
$$




$$
=\max \left\{\begin{array}{c}
\rho\left(\mathfrak{R}_{n(k)}, \mathfrak{R}_{m(k)}\right), \rho\left(\mathfrak{R}_{n(k)+1}, \mathfrak{R}_{n(k)}\right), \\
\rho\left(\mathfrak{R}_{m(k)+1}, \mathfrak{R}_{m(k)}\right), \\
\frac{\rho\left(\mathfrak{R}_{n(k)+1}, \mathfrak{R}_{m(k)}\right)+\rho\left(\mathfrak{R}_{m(k)+1}, \mathfrak{R}_{n(k)}\right)}{2}, \\
\frac{\left(1+\rho\left(\mathfrak{R}_{n(k)+1}, \mathfrak{R}_{n(k)}\right)\right) \rho\left(\mathfrak{R}_{m(k)+1}, \mathfrak{R}_{m(k)}\right)}{1+\rho\left(\mathfrak{R}_{n(k)}, \mathfrak{R}_{m(k)}\right)}
\end{array}\right\} .
$$
obtain

Taking $k \longrightarrow \infty$ and using (30), (32), (36), and (37), we

$$
\lim _{k \longrightarrow \infty} A\left(r_{n(k)}, r_{m(k)}\right) \leq \varepsilon .
$$

Thus, from ( $\left.\Theta^{\prime} 3\right),(37),(40),(43)$, and (45), we have

$$
\begin{aligned}
\theta(\varepsilon) & =\theta\left(\lim _{k \longrightarrow \infty} \rho\left(\Re_{n(k)+1}, \mathfrak{R}_{m(k)+1}\right)\right) \\
& \leq\left[\theta\left(\lim _{k \longrightarrow \infty} A\left(r_{n(k)}, r_{m(k)}\right)\right)\right]^{E\left(\lim _{k \rightarrow \infty} G\left(r_{n(k)}, r_{m(k)}\right)\right)} \\
& \leq[\theta(\varepsilon)]^{z}<\theta(\varepsilon),
\end{aligned}
$$

which is a contradiction. Thus, $\left\{\mathfrak{R}_{n}\right\}$ is a Cauchy sequence. Since $\chi$ is a complete metric space, there exists $r^{*} \in \chi$ such that $\lim _{n \longrightarrow \infty} \rho\left(\Re_{n}, r^{*}\right)=0$ and

$$
\begin{aligned}
\lim _{n \longrightarrow \infty} f\left(r_{2 n}\right) & =\lim _{n \longrightarrow \infty} \breve{T}\left(r_{2 n+1}\right)=\lim _{n \longrightarrow \infty} g\left(r_{2 n+1}\right) \\
& =\lim _{n \longrightarrow \infty} \widehat{S}\left(r_{2 n+2}\right)=r^{*} .
\end{aligned}
$$

Now, we shall prove that $\mathfrak{R}^{*}$ is a common fixed point of $f, g, \widehat{S}$, and $\widetilde{T}$. As $\widehat{S}$ is continuous, so

$$
\lim _{n \longrightarrow \infty} \widehat{S}\left(f\left(r_{2 n}\right)\right)=\widehat{S}\left(r^{*}\right)=\lim _{n \longrightarrow \infty} \widehat{S}\left(\widehat{S}\left(r_{2 n+2}\right)\right) .
$$

Since $(f, \widehat{S})$ is a compatible pair,

$$
\lim _{n \longrightarrow \infty} \rho\left(g\left(\breve{T}\left(r_{2 n+1}\right)\right), \breve{T}\left(g\left(r_{2 n+1}\right)\right)\right)=0 .
$$

From Lemma 1, we have

$$
\lim _{n \longrightarrow \infty} f\left(\widehat{S}\left(r_{2 n}\right)\right)=\widehat{S}\left(r^{*}\right) .
$$

Put $r_{1}=\widehat{S}\left(r_{2 n}\right)$ and $r_{2}=r_{2 n+1}$ in (12) and if $\rho\left(\widehat{S}\left(r^{*}\right), r^{*}\right)>0$, we obtain

$$
\frac{1}{2} \rho\left(f\left(\widehat{S}\left(r_{2 n}\right)\right), \widehat{S}\left(\widehat{S}\left(r_{2 n}\right)\right)\right)<\rho\left(\widehat{S}\left(\widehat{S}\left(r_{2 n}\right)\right), \breve{T}\left(r_{2 n+1}\right)\right) .
$$

Hence,

$$
\Upsilon\left(\rho\left(f\left(\widehat{S}\left(r_{2 n}\right)\right), \widehat{S}\left(\widehat{S}\left(r_{2 n}\right)\right)\right), \rho\left(\widehat{S}\left(\widehat{S}\left(r_{2 n}\right)\right), \breve{T}\left(r_{2 n+1}\right)\right)\right)<0 \text {, }
$$

and by (12), we obtain

$$
\theta\left(\rho\left(f\left(\widehat{S}\left(r_{2 n}\right)\right), g\left(r_{2 n+1}\right)\right)\right) \leq\left[\theta\left(A\left(r_{2 n}, r_{2 n+1}\right)\right)\right]^{E\left(G\left(r_{2 n}, r_{2 n+1}\right)\right)},
$$

where

$$
A\left(r_{2 n}, r_{2 n+1}\right)=\max \left\{\begin{array}{c}
\rho\left(\widehat{S}\left(\widehat{S}\left(r_{2 n}\right)\right), \breve{T}\left(r_{2 n+1}\right)\right), \rho\left(f\left(\widehat{S}\left(r_{2 n}\right)\right), \widehat{S}\left(\widehat{S}\left(r_{2 n}\right)\right)\right), \\
\rho\left(g\left(r_{2 n+1}\right), \breve{T}\left(r_{2 n+1}\right)\right), \\
\frac{\rho\left(f\left(\widehat{S}\left(r_{2 n}\right)\right), \breve{T}\left(r_{2 n+1}\right)\right)+\rho\left(g\left(r_{2 n+1}\right), \widehat{S}\left(\widehat{S}\left(r_{2 n}\right)\right)\right)}{2} \\
\frac{\left(1+\rho\left(f\left(\widehat{S}\left(r_{2 n}\right)\right), \widehat{S}\left(\widehat{S}\left(r_{2 n}\right)\right)\right)\right) \rho\left(g\left(r_{2 n+1}\right), \breve{T}\left(r_{2 n+1}\right)\right)}{1+\rho\left(\widehat{S}\left(\widehat{S}\left(r_{2 n}\right)\right), \breve{T}\left(r_{2 n+1}\right)\right)}
\end{array}\right\},
$$




$$
G\left(r_{2 n}, r_{2 n+1}\right)=\left\{\begin{array}{l}
\rho\left(f\left(\widehat{S}\left(r_{2 n}\right)\right), \widehat{S}\left(\widehat{S}\left(r_{2 n}\right)\right)\right), \rho\left(g\left(r_{2 n+1}\right), \breve{T}\left(r_{2 n+1}\right)\right), \\
\rho\left(f\left(\widehat{S}\left(r_{2 n}\right)\right), \breve{T}\left(r_{2 n+1}\right)\right), \rho\left(g\left(r_{2 n+1}\right), \widehat{S}\left(\widehat{S}\left(r_{2 n}\right)\right)\right)
\end{array}\right\} .
$$

Setting the upper limit in (53), we obtain

$$
\theta\left(\rho\left(\widehat{S}\left(r^{*}\right), r^{*}\right)\right) \leq\left[\theta\left(\rho\left(\widehat{S}\left(r^{*}\right), r^{*}\right)\right)\right]^{z}<\theta\left(\rho\left(\widehat{S}\left(r^{*}\right), r^{*}\right)\right),
$$

a contradiction. Thus, $\rho\left(\widehat{S}\left(r^{*}\right), r^{*}\right)=0$ and $\widehat{S}\left(r^{*}\right)=r^{*}$. Again since $T$ is continuous,

$$
\lim _{n \longrightarrow \infty} \breve{T}\left(g\left(r_{2 n+1}\right)\right)=\breve{T}\left(r^{*}\right)=\lim _{n \longrightarrow \infty} \breve{T}\left(\breve{T}\left(r_{2 n+1}\right)\right) .
$$

Since $(g, \breve{T})$ is a compatible pair,

$$
\lim _{n \longrightarrow \infty} \rho\left(g\left(\breve{T}\left(r_{2 n+1}\right)\right), \breve{T}\left(g\left(r_{2 n+1}\right)\right)\right)=0 .
$$

From Lemma 1, we have

$$
\lim _{n \longrightarrow \infty} g\left(\breve{T}\left(r_{2 n+1}\right)\right)=\breve{T}\left(r^{*}\right) .
$$

Put $r_{1}=r_{2 n}$ and $r_{2}=\breve{T}\left(r_{2 n+1}\right)$ in (12) and if $\rho\left(r^{*}, T\left(r^{*}\right)\right)>0$, we obtain

$$
\frac{1}{2} \rho\left(f\left(r_{2 n}\right), \widehat{S}\left(r_{2 n}\right)\right)<\rho\left(\widehat{S}\left(r_{2 n}\right), \breve{T}\left(\breve{T}\left(r_{2 n+1}\right)\right)\right) .
$$

Therefore,

$$
\Upsilon\left(\begin{array}{c}
\rho\left(f\left(r_{2 n}\right), \widehat{S}\left(r_{2 n}\right)\right), \\
\rho\left(\widehat{S}\left(r_{2 n}\right), \breve{T}\left(\breve{T}\left(r_{2 n+1}\right)\right)\right)
\end{array}\right)<0 .
$$

By (12), one obtains

$$
\theta\left(\rho\left(f\left(r_{2 n}\right), g\left(\breve{T}\left(r_{2 n+1}\right)\right)\right)\right) \leq\left[\theta\left(A\left(r_{2 n}, r_{2 n+1}\right)\right)\right]^{E\left(G\left(r_{2 n}, r_{2 n+1}\right)\right)},
$$

where

$$
A\left(r_{2 n}, r_{2 n+1}\right)=\max \left\{\begin{array}{c}
\rho\left(\widehat{S}\left(r_{2 n}\right), \breve{T}\left(\breve{T}\left(r_{2 n+1}\right)\right)\right), \rho\left(f\left(r_{2 n}\right), \widehat{S}\left(r_{2 n}\right)\right), \\
\rho\left(g\left(\breve{T}\left(r_{2 n+1}\right)\right), \breve{T}\left(\breve{T}\left(r_{2 n+1}\right)\right)\right), \\
\frac{\left.\left(r_{2 n}\right), \breve{T}\left(\breve{T}\left(r_{2 n+1}\right)\right)\right)+\rho\left(g\left(\breve{T}\left(r_{2 n+1}\right)\right), \widehat{S}\left(r_{2 n}\right)\right)}{2}, \\
\frac{\left(1+\rho\left(f\left(r_{2 n}\right), \widehat{S}\left(r_{2 n}\right)\right)\right) \rho\left(g\left(\breve{T}\left(r_{2 n+1}\right)\right), \breve{T}\left(\breve{T}\left(r_{2 n+1}\right)\right)\right.}{1+\rho\left(\widehat{S}\left(r_{2 n}\right), \breve{T}\left(\breve{T}\left(r_{2 n+1}\right)\right)\right)} \\
\rho\left(r_{2 n}, r_{2 n+1}\right)=\left\{\begin{array}{c}
\left.\rho\left(r_{2 n}\right), \widehat{S}\left(r_{2 n}\right)\right), \rho\left(g\left(\breve{T}\left(r_{2 n+1}\right)\right), \breve{T}\left(\breve{T}\left(r_{2 n+1}\right)\right)\right), \\
\left.f\left(r_{2 n}\right), \breve{T}\left(\breve{T}\left(r_{2 n+1}\right)\right)\right), \rho\left(g\left(\breve{T}\left(r_{2 n+1}\right)\right), \widehat{S}\left(r_{2 n}\right)\right)
\end{array}\right\} . \\
\frac{1}{2} \rho\left(\widehat{S}\left(r^{*}\right), \breve{T}\left(r_{2 n+1}\right)\right)<\rho\left(f\left(r^{*}\right), \widehat{S}\left(r^{*}\right)\right) .
\end{array}\right\}
$$

Setting the upper limit, we obtain

$\theta\left(\rho\left(r^{*}, \breve{T}\left(r^{*}\right)\right)\right) \leq\left[\theta\left(\rho\left(r^{*}, \breve{T}\left(r^{*}\right)\right)\right)\right]^{z}<\theta\left(\rho\left(r^{*}, \breve{T}\left(r^{*}\right)\right)\right)$,

a contradiction. Thus, $\rho\left(r^{*}, \breve{T}\left(r^{*}\right)\right)=0$ and so $r^{*}=\breve{T}\left(r^{*}\right)$. Suppose $\rho\left(f\left(r^{*}\right), r^{*}\right)>0$, we obtain
Hence,

$$
\Upsilon\left(\begin{array}{c}
\rho\left(f\left(r^{*}\right), \widehat{S}\left(r^{*}\right)\right), \\
\rho\left(\widehat{S}\left(r^{*}\right), \breve{T}\left(r_{2 n+1}\right)\right)
\end{array}\right)<0,
$$


and by (12), we obtain

where

$$
\theta\left(\rho\left(f\left(r^{*}\right), g\left(r_{2 n+1}\right)\right)\right) \leq\left[\theta\left(A\left(r^{*}, r_{2 n+1}\right)\right)\right]^{E\left(G\left(r^{*}, r_{2 n+1}\right)\right)},
$$

$$
A\left(r^{*}, r_{2 n+1}\right)=\max \left\{\begin{array}{c}
\rho\left(\widehat{S}\left(r^{*}\right), \breve{T}\left(r_{2 n+1}\right)\right), \rho\left(f\left(r^{*}\right), \widehat{S}\left(r^{*}\right)\right), \\
\rho\left(g\left(r_{2 n+1}\right), \breve{T}\left(r_{2 n+1}\right)\right), \frac{\rho\left(f\left(r^{*}\right), \breve{T}\left(r_{2 n+1}\right)\right)+\rho\left(g\left(r_{2 n+1}\right), \widehat{S}\left(r^{*}\right)\right)}{2}, \\
\frac{\left(1+\rho\left(f\left(r^{*}\right), \widehat{S}\left(r^{*}\right)\right)\right) \rho\left(g\left(r_{2 n+1}\right), \breve{T}\left(r_{2 n+1}\right)\right)}{1+\rho\left(\widehat{S}\left(r^{*}\right), \breve{T}\left(r_{2 n+1}\right)\right)} \\
G\left(r^{*}, r_{2 n+1}\right)=\left\{\begin{array}{c}
\rho\left(f\left(r^{*}\right), \widehat{S}\left(r^{*}\right)\right), \rho\left(g\left(r_{2 n+1}\right), \breve{T}\left(r_{2 n+1}\right)\right), \\
\rho\left(f\left(r^{*}\right), \breve{T}\left(r_{2 n+1}\right)\right), \rho\left(g\left(r_{2 n+1}\right), \widehat{S}\left(r^{*}\right)\right)
\end{array}\right\} .
\end{array}\right.
$$

Taking the upper limit in (66), and as $r^{*}=\widehat{T}\left(r^{*}\right)=\widehat{S}\left(r^{*}\right)$, so we obtain

$$
\theta\left(\rho\left(f\left(r^{*}\right), r^{*}\right)\right) \leq\left[\theta\left(\rho\left(f\left(r^{*}\right), r^{*}\right)\right)\right]^{z}<\theta\left(\rho\left(f\left(r^{*}\right), r^{*}\right)\right),
$$

a contradiction. Thus, $\rho\left(f\left(r^{*}\right), r^{*}\right)=0$ and $r^{*}=f\left(r^{*}\right)$. Finally, suppose $\rho\left(r^{*}, g\left(r^{*}\right)\right)>0$, and as $r^{*}=\breve{T}\left(r^{*}\right)=$ $\widehat{S}\left(r^{*}\right)=f\left(r^{*}\right)$, so, we obtain

$$
\Upsilon\left(0, \rho\left(r^{*}, g\left(r^{*}\right)\right)\right)<0 .
$$

Thus, from (12)

$$
\begin{aligned}
\theta\left(\rho\left(r^{*}, g\left(r^{*}\right)\right)\right) & =\theta\left(\rho\left(f\left(r^{*}\right), g\left(r^{*}\right)\right)\right) \leq\left[\theta\left(\rho\left(r^{*}, g\left(r^{*}\right)\right)\right)\right]^{z} \\
& <\theta\left(\rho\left(r^{*}, g\left(r^{*}\right)\right)\right),
\end{aligned}
$$

a contradiction. Thus, $\rho\left(r^{*}, g\left(r^{*}\right)\right)=0$ and $r^{*}=g\left(r^{*}\right)$. Therefore, $r^{*}$ is a common of the four mappings $\widehat{S}, g, f$ and $T$. Next, assume that $v^{*}$ is another common of the four mappings $\widehat{S}, g, f$, and $\breve{T}$ such that $r^{*} \neq v^{*}$, one obtains

$$
\Upsilon\left(0, \rho\left(\widehat{S}\left(r^{*}\right), \breve{T}\left(v^{*}\right)\right)\right)<0 \text {. }
$$

Then, by (12), we obtain

$$
\theta\left(\rho\left(r^{*}, v^{*}\right)\right)=\theta\left(\rho\left(f\left(r^{*}\right), g\left(v^{*}\right)\right)\right) \leq\left[\theta\left(A\left(r^{*}, v^{*}\right)\right)\right]^{E\left(G\left(r^{*}, v^{*}\right)\right)},
$$

where

$$
\begin{gathered}
A\left(r^{*}, v^{*}\right)=\max \left\{\begin{array}{c}
\rho\left(\widehat{S}\left(r^{*}\right), \breve{T}\left(v^{*}\right)\right), \rho\left(f\left(r^{*}\right), \widehat{S}\left(r^{*}\right)\right), \rho\left(g\left(v^{*}\right), \breve{T}\left(v^{*}\right)\right), \\
\left.\frac{\rho\left(f\left(r^{*}\right), \breve{T}\left(v^{*}\right)\right)+\rho\left(g\left(v^{*}\right), \widehat{S}\left(r^{*}\right)\right)}{2}, \frac{\left(1+\rho\left(f\left(r^{*}\right), \widehat{S}\left(r^{*}\right)\right)\right) \rho\left(g\left(v^{*}\right), \breve{T}\left(v^{*}\right)\right)}{1+\rho\left(\widehat{S}\left(r^{*}\right), \breve{T}\left(v^{*}\right)\right)}\right\},
\end{array}\right. \\
G\left(r^{*}, v^{*}\right)=\left\{\begin{array}{l}
\rho\left(f\left(r^{*}\right), \widehat{S}\left(r^{*}\right)\right), \rho\left(g\left(v^{*}\right), \breve{T}\left(v^{*}\right)\right), \\
\rho\left(f\left(r^{*}\right), \breve{T}\left(v^{*}\right)\right), \rho\left(g\left(v^{*}\right), \widehat{S}\left(r^{*}\right)\right)
\end{array}\right\} .
\end{gathered}
$$


It implies that

$$
\theta\left(\rho\left(r^{*}, v^{*}\right)\right) \leq\left[\theta\left(\rho\left(r^{*}, v^{*}\right)\right)\right]^{z}<\theta\left(\rho\left(r^{*}, v^{*}\right)\right),
$$

a contradiction. Thus, $r^{*}=v^{*}$. Therefore, $r^{*}$ is the unique common fixed point of $\widehat{S}, g, f$, and $\widehat{T}$.

Corollary 1. Let $(\chi, \rho)$ be a complete metric space, and $f, g, \widehat{S}, \breve{T}: \chi \longrightarrow \chi$ be four single-valued maps. Suppose that if, for all $r, j \in \chi$ with $\rho(f r, g j)>0$ for some $\theta \in \mu, z \in[0,1)$ and $Y \in \Phi$,

$$
\Upsilon(\rho(f r, \widehat{S} r), \rho(\widehat{S} r, \breve{T} j))<0 \Longrightarrow \theta(\rho(f r, g j)) \leq[\theta(A(r, j))]^{z},
$$

where

$$
A(r, j)=\max \left\{\begin{array}{c}
\rho(\widehat{S} r, \breve{T} j), \rho(f r, \widehat{S} r), \rho(g j, \breve{T} j), \frac{\rho(f r, \breve{T} j)+\rho(g j, \widehat{S} r)}{2}, \\
\frac{(1+\rho(f r, \widehat{S} r)) \rho(g j, \breve{T} j)}{1+\rho(\widehat{S} r, \breve{T} j)}
\end{array}\right\},
$$

with $f(\chi) \subseteq \breve{T}(\chi)$ and $g(\chi) \subseteq \widehat{S}(\chi)$. If $(f, \widehat{S})$ and $(g, \breve{T})$ are compatible pairs and $\widehat{T}$ and $\widehat{S}$ are continuous on $(\chi, \rho)$, then $f, g, \widehat{S}$, and $T$ have a unique common fixed point in $\chi$.

Example 5. Let $\chi=[0,1]$ and define the function $\rho: \chi \times \chi \longrightarrow[0,+\infty)$ by $\rho(r, j)=|r-j|$. Clearly, $(\chi, \rho)$ is a complete metric space. Let $\theta(t)=e^{t}, \quad t>0$, and $\Upsilon(s, t)=(s / 2)-t$, then $\theta \in \mu$ and $\Upsilon \in \Phi$. Define the mappings $\widehat{S}, g, f, T: \chi \longrightarrow \chi$ by

$$
\begin{aligned}
& \widehat{S}(r)=\left(\frac{r}{3}\right)^{8}, \\
& g(r)=\left(\frac{r}{3}\right)^{8}, \\
& f(r)=\left(\frac{r}{3}\right)^{16}, \\
& \breve{T}(r)=\left(\frac{r}{3}\right)^{4} .
\end{aligned}
$$

Clearly, $f(\chi) \subseteq \breve{T}(\chi)$ and $g(\chi) \subseteq \widehat{S}(\chi)$, if $\left\{r_{n}\right\}$ is a sequence in $\chi$ such that

$$
\lim _{n \longrightarrow \infty} f\left(r_{n}\right)=\lim _{n \longrightarrow \infty} \widehat{S}\left(r_{n}\right)=t, \quad \text { for some } t \in \chi,
$$

then

$$
\lim _{n \longrightarrow \infty}\left|f\left(r_{n}\right)-t\right|=\lim _{n \longrightarrow \infty}\left|\widehat{S}\left(k_{n}\right)-t\right|=0,
$$

and equivalently,

$$
\lim _{n \longrightarrow \infty}\left|\left[\frac{r_{n}}{3}\right]^{16}-t\right|=\lim _{n \longrightarrow \infty}\left|\left[\frac{r_{n}}{3}\right]^{8}-t\right|=0 .
$$

Thus,

$$
\lim _{n \longrightarrow \infty}\left|\left[r_{n}\right]^{16}-3^{16} t\right|=\lim _{n \longrightarrow \infty}\left|\left[r_{n}\right]^{8}-3^{8} t\right|=0 .
$$

We conclude $t^{1 / 16}=t^{1 / 8}$ (by uniqueness of limit); hence, $t \in\{0,1\}$. Using continuity of $f$ and $\widehat{S}$, one obtains

$$
\begin{aligned}
\lim _{n \longrightarrow \infty} \rho\left(f \widehat{S}\left(r_{n}\right), \widehat{S} f\left(r_{n}\right)\right) & =\lim _{n \longrightarrow \infty}\left|f \widehat{S}\left(r_{n}\right)-\widehat{S} f\left(r_{n}\right)\right| \\
& =|f(t)-\widehat{S}(t)|=|0-0|=0,
\end{aligned}
$$

for $t=0 \in \chi$. Hence, the pair $(f, \widehat{S})$ is compatible. Similarly, the pair $(g, T)$ is compatible. Define $E: \mathbb{R}^{4} \longrightarrow \mathbb{R}^{+}$as $E\left(s_{1}, s_{2}, s_{3}, s_{4}\right)=(9 / 10)$. Next, for all $r, j \in \chi$ with $\rho(f r, g j)>0$,

$$
\Upsilon(\rho(f r, \widehat{S} r), \rho(\widehat{S} r, \breve{T} j))<0,
$$

and so

$$
\theta(\rho(f r, g j)) \leq[\theta(A(r, j))]^{E(G(r, j))} .
$$

Hence, all hypotheses of Theorem 2 are satisfied. Thus, $f, g, \widehat{S}$, and $\widetilde{T}$ have a unique common fixed point, which is $r^{*}=0$.

We can easily obtain the following results by chasing the proof of Theorem 2 .

Corollary 2. Let $(\chi, \rho)$ be a complete metric space, and $f, g, \widehat{S}, T: \chi \longrightarrow \chi$ be four single-valued maps. Suppose that if, for all $r, j \in \chi$, with $\rho(f r, g j)>0$, for some $\theta \in \mu$, $\Upsilon \in \Phi$ and $E: \mathbb{R}^{+4} \longrightarrow \mathbb{R}^{+}$,

$$
\begin{aligned}
\Upsilon(\rho(f r, \widehat{S} r), \rho(\widehat{S} r, \breve{T} j)) & <0 \Longrightarrow \theta(\rho(f r, g j)) \\
& \leq[\theta(\max \{\rho(f r, \widehat{S} r), \rho(g j, \breve{T} j)\})]^{E(G(r, j))},
\end{aligned}
$$

where

$$
G(r, j)=\{\rho(f r, \widehat{S} r), \rho(g j, \breve{T} j), \rho(f r, \breve{T} j), \rho(g j, \widehat{S} r)\},
$$

with $f(\chi) \subseteq \breve{T}(\chi)$ and $g(\chi) \subseteq \widehat{S}(\chi)$. If $(f, \widehat{S})$ and $(g, \breve{T})$ are compatible pairs, and $T$ and $\widehat{S}$ are continuous on $(\chi, \rho)$, then $f, g, \widehat{S}$, and $T$ have a unique common fixed point in $\chi$. 
The following corollary generalizes the result introduced by Sehgal [34].

Corollary 3. Let $(\chi, \rho)$ be a complete metric space, and $f, g, \widehat{S}, \widehat{T}: \chi \longrightarrow \chi$ be four single-valued maps. Suppose that if, for all $r, j \in \chi$, with $\rho(f r, g j)>0$, for some $\theta \in \mu, \Upsilon \in \Phi$ and $E: \mathbb{R}^{+4} \longrightarrow \mathbb{R}^{+}, \Upsilon(\rho(f r, \widehat{S} r), \rho(\widehat{S} r, T j))<0$ implies that $\theta(\rho(f r, g j)) \leq[\theta(\max \{\rho(\widehat{S} r, \breve{T} j), \rho(f r, \widehat{S} r), \rho(g j, \breve{T} j)\})]^{E(G(r, j))}$,

where

$$
G(r, j)=\{\rho(f r, \widehat{S} r), \rho(g j, \breve{T} j), \rho(f r, \breve{T} j), \rho(g j, \widehat{S} r)\},
$$

with $f(\chi) \subseteq \breve{T}(\chi)$ and $g(\chi) \subseteq \widehat{S}(\chi)$. If $(f, \widehat{S})$ and $(g, \breve{T})$ are compatible pairs, and $T$ and $\widehat{S}$ are continuous on $(\chi, \rho)$, then $f, g, \widehat{S}$, and $\widetilde{T}$ have a unique common fixed point in $\chi$.

\section{On Suzuki-Type Ćirić JS-Contractions}

We start this section with the following definition.

Definition 4. Let $(\chi, \rho)$ be a metric space and $f, g, \widehat{S}, T: \chi \longrightarrow \chi$ be four single-valued maps. These maps form a new generalized Suzuki-type Ćirić JS-contraction if, for all $r, j \in \mathcal{X}$, for some $\theta \in \Psi, \quad \Upsilon \in \Phi$, $\Upsilon(\rho(f r, \widehat{S} r), \rho(\widehat{S} r, T j))<0$ implies that

$$
\begin{aligned}
\theta(\rho(f r, g j)) \leq & {[\theta(\rho(\widehat{S} r, \breve{T} j))]^{a} \cdot[\theta(\rho(f r, \widehat{S} r))]^{b} } \\
& \cdot[\theta(\rho(g j, \breve{T} j))]^{c} \cdot[\theta(\rho(f r, \breve{T} j)+\rho(g j, \widehat{S} r))]^{d},
\end{aligned}
$$

where $a, b, c, d \geq 0$ with $a+b+c+2 d<1$.

Our second result is as follows.
Theorem 3. Let $(\chi, \rho)$ be a complete metric space and $f, g, \widehat{S}, \breve{T}: \chi \longrightarrow \chi$ be four single-valued maps. Suppose that the mappings $f, g, \widehat{S}, T$ form a generalized Suzuki-type Ćiric JS-contraction with $f(\chi) \subseteq T(\chi)$ and $g(\chi) \subseteq \widehat{S}(\chi)$. If $(f, \widehat{S})$ and $(g, \widehat{T})$ are compatible pairs and $\widehat{T}$ and $\widehat{S}$ are continuous on $(\chi, \rho)$, then $f, g, \widehat{S}$, and $T$ have a unique common fixed point in $\chi$.

Proof. Let $r_{0} \in \chi$ be an arbitrary point. As $f(\chi) \subseteq \breve{T}(\chi)$, there exists $r_{0} \in \chi$ such that

$$
f\left(r_{0}\right)=\breve{T}\left(r_{1}\right) .
$$

Since $g\left(r_{1}\right) \in \widehat{S}(\chi)$, we can choose $r_{2} \in \chi$ such that $g\left(r_{1}\right)=\widehat{S}\left(r_{2}\right)$. In general, $r_{2 n+1}$ and $r_{2 n+2}$ are chosen in $\chi$ such that

$$
\begin{aligned}
f\left(r_{2 n}\right) & =\breve{T}\left(r_{2 n+1}\right), \\
g\left(r_{2 n+1}\right) & =\widehat{S}\left(r_{2 n+2}\right) .
\end{aligned}
$$

We construct a sequence $\left\{\mathfrak{R}_{n}\right\}$ in $\chi$ such that

$$
\begin{gathered}
\mathfrak{R}_{2 n}=f\left(r_{2 n}\right)=\breve{T}\left(r_{2 n+1}\right), \\
\mathfrak{R}_{2 n+1}=g\left(r_{2 n+1}\right)=\widehat{S}\left(r_{2 n+2}\right),
\end{gathered}
$$

for $n=0,1,2, \ldots$ Since

$$
\begin{aligned}
\frac{1}{2} \rho\left(f\left(r_{2 n}\right), \widehat{S}\left(r_{2 n}\right)\right) & =\frac{1}{2} \rho\left(\mathfrak{R}_{2 n}, \mathfrak{R}_{2 n-1}\right)<\rho\left(\mathfrak{R}_{2 n-1}, \mathfrak{R}_{2 n}\right) \\
& =\rho\left(\widehat{S}\left(r_{2 n}\right), \breve{T}\left(r_{2 n+1}\right)\right),
\end{aligned}
$$

we have

$$
\Upsilon\left(\rho\left(f\left(r_{2 n}\right), \widehat{S}\left(r_{2 n}\right)\right), \rho\left(\widehat{S}\left(r_{2 n}\right), \breve{T}\left(r_{2 n+1}\right)\right)\right)<0 .
$$

Then, from (89),

$$
\begin{aligned}
\theta\left(\rho\left(\mathfrak{R}_{2 n}, \mathfrak{R}_{2 n+1}\right)\right)= & \theta\left(\rho\left(f\left(r_{2 n}\right), g\left(r_{2 n+1}\right)\right)\right) \leq\left[\theta\left(\rho\left(\widehat{S}\left(r_{2 n}\right), \check{T}\left(r_{2 n+1}\right)\right)\right)\right]^{a} \cdot\left[\theta\left(\rho\left(f\left(r_{2 n}\right), \widehat{S}\left(r_{2 n}\right)\right)\right)\right]^{b} \\
& \cdot\left[\theta\left(\rho\left(g\left(r_{2 n+1}\right), \check{T}\left(r_{2 n+1}\right)\right)\right)\right]^{c} \cdot\left[\theta\left(\rho\left(f\left(r_{2 n}\right), \check{T}\left(r_{2 n+1}\right)\right)+\rho\left(g\left(r_{2 n+1}\right), \widehat{S}\left(r_{2 n}\right)\right)\right)\right]^{d} \\
= & {\left[\theta\left(\rho\left(\mathfrak{R}_{2 n-1}, \mathfrak{R}_{2 n}\right)\right)\right]^{a} \cdot\left[\theta\left(\rho\left(\mathfrak{R}_{2 n}, \mathfrak{R}_{2 n-1}\right)\right)\right]^{b} \cdot\left[\theta\left(\rho\left(\mathfrak{R}_{2 n+1}, \mathfrak{R}_{2 n}\right)\right)\right]^{c} \cdot\left[\theta\left(\rho\left(\mathfrak{R}_{2 n}, \mathfrak{R}_{2 n}\right)+\rho\left(\mathfrak{R}_{2 n+1}, \mathfrak{R}_{2 n-1}\right)\right)\right]^{d} } \\
= & {\left[\theta\left(\rho\left(\mathfrak{R}_{2 n-1}, \mathfrak{R}_{2 n}\right)\right)\right]^{a+b} \cdot\left[\theta\left(\rho\left(\mathfrak{R}_{2 n+1}, \mathfrak{R}_{2 n}\right)\right)\right]^{c} \cdot\left[\theta\left(\rho\left(\mathfrak{R}_{2 n-1}, \mathfrak{R}_{2 n}\right)+\rho\left(\mathfrak{R}_{2 n}, \mathfrak{R}_{2 n+1}\right)\right)\right]^{d} . }
\end{aligned}
$$

By $\left(\Psi_{5}\right)$, we have, for every $n \in \mathbb{N}$,

$$
\begin{aligned}
\theta\left(\rho\left(\mathfrak{R}_{2 n-1}, \mathfrak{R}_{2 n}\right)+\rho\left(\mathfrak{R}_{2 n}, \mathfrak{R}_{2 n+1}\right)\right) \leq & \theta\left(\rho\left(\mathfrak{R}_{2 n-1}, \mathfrak{R}_{2 n}\right)\right) \\
& \cdot \theta\left(\rho\left(\boldsymbol{R}_{2 n}, \mathfrak{R}_{2 n+1}\right)\right) .
\end{aligned}
$$

$$
\begin{aligned}
1<\theta\left(\rho\left(\mathfrak{R}_{2 n}, \mathfrak{R}_{2 n+1}\right)\right) \leq & {\left[\theta\left(\rho\left(\mathfrak{R}_{2 n-1}, \mathfrak{R}_{2 n}\right)\right)\right]^{a+b+d} } \\
\cdot & {\left[\theta\left(\rho\left(\mathfrak{R}_{2 n+1}, \mathfrak{R}_{2 n}\right)\right)\right]^{c+d} . }
\end{aligned}
$$

It implies that

$$
\left[\theta\left(\rho\left(\mathfrak{R}_{2 n}, \mathfrak{R}_{2 n+1}\right)\right)\right]^{1-c-d} \leq\left[\theta\left(\rho\left(\boldsymbol{R}_{2 n-1}, \mathfrak{R}_{2 n}\right)\right)\right]^{a+b+d} \text {. }
$$

Hence, (95) becomes

Therefore, 


$$
\theta\left(\rho\left(\mathfrak{R}_{2 n}, \mathfrak{R}_{2 n+1}\right)\right) \leq\left[\theta\left(\rho\left(\mathfrak{R}_{2 n-1}, \mathfrak{R}_{2 n}\right)\right)\right]^{(a+b+d) /(1-c-d)},
$$

and so

$$
\theta\left(\rho\left(\mathfrak{R}_{n}, \mathfrak{R}_{n+1}\right)\right) \leq\left[\theta\left(\rho\left(\mathfrak{R}_{n-1}, \mathfrak{R}_{n}\right)\right)\right]^{(a+b+d) /(1-c-d)} .
$$

It implies that

$$
\begin{aligned}
1 & <\theta\left(\rho\left(\mathfrak{R}_{n}, \mathfrak{R}_{n+1}\right)\right) \\
& \leq\left[\theta\left(\rho\left(\mathfrak{R}_{n-1}, \mathfrak{R}_{n}\right)\right)\right]^{((a+b+d) /(1-c-d))^{2}} \\
& \vdots \\
& \leq\left[\theta\left(\rho\left(\mathfrak{R}_{0}, \mathfrak{R}_{1}\right)\right)\right]^{((a+b+d) /(1-c-d))^{n}},
\end{aligned}
$$

for all $n \in \mathbb{N}$. Taking the limit as $n \longrightarrow \infty$ in (101) and knowing that $\theta \in \Psi$, we have

$$
\lim _{n \longrightarrow \infty} \theta\left(\rho\left(\mathfrak{R}_{n}, \mathfrak{R}_{n+1}\right)\right)=1 .
$$

By $\left(\Psi_{3}\right)$, we obtain

$$
\lim _{n \longrightarrow \infty} \rho\left(\Re_{n}, \mathfrak{R}_{n+1}\right)=0 .
$$

From condition $\left(\Psi_{4}\right)$, there exist $q \in(0,1)$ and $\ell \in(0, \infty]$ such that

$$
\lim _{n \longrightarrow \infty} \frac{\theta\left(\rho\left(\mathfrak{R}_{n}, \mathfrak{R}_{n+1}\right)\right)-1}{\left[\rho\left(\mathfrak{R}_{n}, \mathfrak{R}_{n+1}\right)\right]^{q}}=\ell .
$$

Suppose that $\ell<\infty$. Let $V=(\ell / 2)>0$. From the definition of the limit, there exists $n_{0} \in \mathbb{N}$ such that

$$
\left|\frac{\theta\left(\rho\left(\mathfrak{R}_{n}, \mathfrak{R}_{n+1}\right)\right)-1}{\left[\rho\left(\mathfrak{R}_{n}, \mathfrak{R}_{n+1}\right)\right]^{q}}-\ell\right| \leq V, \quad \text { for all } n \geq n_{0} .
$$

This implies

$$
\frac{\theta\left(\rho\left(\mathfrak{R}_{n}, \mathfrak{R}_{n+1}\right)\right)-1}{\left[\rho\left(\mathfrak{R}_{n}, \mathfrak{R}_{n+1}\right)\right]^{q}} \geq \ell-V=V, \quad \text { for all } n \geq n_{0} .
$$

Then,

$$
n\left[\rho\left(\mathfrak{R}_{n}, \mathfrak{R}_{n+1}\right)\right]^{q} \leq \operatorname{Pn}\left[\theta\left(\rho\left(\mathfrak{R}_{n}, \Re_{n+1}\right)\right)-1\right], \quad \text { for all } n \geq n_{0},
$$

where $P=(1 / V)$. Suppose now that $\ell=\infty$. Let $V>0$ be an arbitrary positive number. From the definition of the limit, there exists $n_{0} \geq 1$ such that

$$
\frac{\theta\left(\rho\left(\mathfrak{R}_{n}, \mathfrak{R}_{n+1}\right)\right)-1}{\left[\rho\left(\mathfrak{R}_{n}, \mathfrak{R}_{n+1}\right)\right]^{q}} \geq V, \quad \text { for all } n \geq n_{0},
$$

which implies

$$
n\left[\rho\left(\mathfrak{R}_{n}, \mathfrak{R}_{n+1}\right)\right]^{q} \leq \operatorname{Pn}\left[\theta\left(\rho\left(\mathfrak{R}_{n}, \mathfrak{R}_{n+1}\right)\right)-1\right], \quad n \geq n_{0},
$$

where $P=(1 / V)$. Thus, in all cases, there exist $P>0$ and $n_{0} \geq 1$ such that

$$
n\left[\rho\left(\mathfrak{R}_{n}, \mathfrak{R}_{n+1}\right)\right]^{q} \leq \operatorname{Pn}\left[\theta\left(\rho\left(\mathfrak{R}_{n}, \mathfrak{R}_{n+1}\right)\right)-1\right], \quad n \geq n_{0} .
$$

By using (101), we obtain

$$
n\left[\rho\left(\Re_{n}, \mathfrak{R}_{n+1}\right)\right]^{q} \leq \operatorname{Pn}\left(\left[\theta\left(\rho\left(\mathfrak{R}_{0}, \mathfrak{R}_{1}\right)\right)\right]^{((a+b+d) /(1-c-d))^{n}}-1\right),
$$

for all $n \geq n_{0}$.

Setting $n \longrightarrow \infty$ in inequality (111), we obtain

$$
\lim _{n \longrightarrow \infty} n\left[\rho\left(\mathfrak{R}_{n}, \mathfrak{R}_{n+1}\right)\right]^{q}=0 .
$$

Thus, there exists $n_{1} \in \mathbb{N}$ such that

$$
\rho\left(\Re_{n}, \Re_{n+1}\right) \leq \frac{1}{n^{(1 / q)}}, \quad \text { for all } n \geq n_{1} .
$$

To prove $\left\{\Re_{n}\right\}$ is a Cauchy sequence, we use (113) and for $m>n \geq n_{1}$,

$$
\rho\left(\mathfrak{R}_{n}, \mathfrak{R}_{m}\right) \leq \sum_{i=n}^{m-1} \rho\left(\mathfrak{R}_{i}, \mathfrak{R}_{i+1}\right) \leq \sum_{i=n}^{\infty} \rho\left(\mathfrak{R}_{i}, \mathfrak{R}_{i+1}\right) \leq \sum_{i=n}^{\infty} \frac{1}{i^{(1 / q)}} .
$$

The convergence of the series $\sum_{i=n}^{\infty} 1 / i^{(1 / q)}$ entails $\lim _{n, m \longrightarrow \infty} \rho\left(\mathfrak{R}_{n}, \mathfrak{R}_{m}\right)=0$. Thus, $\left\{\mathfrak{R}_{n}\right\}$ is a Cauchy sequence. Since $\chi$ is a complete metric space, there exists $r^{*} \in \chi$ such that $\lim _{n \longrightarrow \infty} d\left(\Re_{n}, r^{*}\right)=0$ and

$$
\begin{aligned}
\lim _{n \longrightarrow \infty} f\left(r_{2 n}\right) & =\lim _{n \longrightarrow \infty} \breve{T}\left(r_{2 n+1}\right)=\lim _{n \longrightarrow \infty} g\left(r_{2 n+1}\right) \\
& =\lim _{n \longrightarrow \infty} \widehat{S}\left(r_{2 n+2}\right)=r^{*} .
\end{aligned}
$$

Now, we shall prove that $\Re^{*}$ is a common fixed point of $f, g, \widehat{S}$, and $T$. As $\widehat{S}$ is continuous, so

$$
\lim _{n \longrightarrow \infty} \hat{S}\left(f\left(r_{2 n}\right)\right)=\widehat{S}\left(r^{*}\right)=\lim _{n \longrightarrow \infty} \widehat{S}\left(\widehat{S}\left(r_{2 n+2}\right)\right) .
$$

Since $(f, \widehat{S})$ is a compatible pair,

$$
\lim _{n \longrightarrow \infty} \rho\left(f\left(\widehat{S}\left(r_{2 n}\right)\right), \widehat{S}\left(f\left(r_{2 n}\right)\right)\right)=0 .
$$

From Lemma 1, we have

$$
\lim _{n \rightarrow \infty} f\left(\widehat{S}\left(r_{2 n}\right)\right)=\widehat{S}\left(r^{*}\right) .
$$

Put $r=\widehat{S}\left(r_{2 n}\right)$ and $j=r_{2 n+1} \quad$ in (89) and if $\rho\left(\widehat{S}\left(r^{*}\right), r^{*}\right)>0$, we obtain

$$
\frac{1}{2} \rho\left(f\left(\widehat{S}\left(r_{2 n}\right)\right), \widehat{S}\left(\widehat{S}\left(r_{2 n}\right)\right)\right)<\rho\left(\widehat{S}\left(\widehat{S}\left(r_{2 n}\right)\right), \breve{T}\left(r_{2 n+1}\right)\right) .
$$

Hence,

$$
\Upsilon\left(\begin{array}{c}
\rho\left(f\left(\widehat{S}\left(r_{2 n}\right)\right), \widehat{S}\left(\widehat{S}\left(r_{2 n}\right)\right)\right), \\
\rho\left(\widehat{S}\left(\widehat{S}\left(r_{2 n}\right)\right), \breve{T}\left(r_{2 n+1}\right)\right)
\end{array}\right)<0 .
$$

Then, from (89), we obtain 


$$
\begin{aligned}
\theta\left(\rho\left(f\left(\widehat{S}\left(r_{2 n}\right)\right), g\left(r_{2 n+1}\right)\right)\right) \leq & {\left[\theta\left(\rho\left(\widehat{S}\left(\widehat{S}\left(r_{2 n}\right)\right), \breve{T}\left(r_{2 n+1}\right)\right)\right)\right]^{a} } \\
& \cdot\left[\theta\left(\rho\left(f\left(\widehat{S}\left(r_{2 n}\right)\right), \widehat{S}\left(\widehat{S}\left(r_{2 n}\right)\right)\right)\right)\right]^{b} \\
& \cdot\left[\theta\left(\rho\left(g\left(r_{2 n+1}\right), \breve{T}\left(r_{2 n+1}\right)\right)\right)\right]^{c} \\
& \cdot\left[\theta \left(\rho\left(f\left(\widehat{S}\left(r_{2 n}\right)\right), \breve{T}\left(r_{2 n+1}\right)\right)\right.\right. \\
& \left.\left.+\rho\left(g\left(r_{2 n+1}\right), \widehat{S}\left(\widehat{S}\left(r_{2 n}\right)\right)\right)\right)\right]^{d} .
\end{aligned}
$$

Setting the upper limit, we obtain

$$
\begin{aligned}
\theta\left(\rho\left(\widehat{S}\left(r^{*}\right), r^{*}\right)\right) \leq & {\left[\theta\left(\rho\left(\widehat{S} r^{*}, r^{*}\right)\right)\right]^{a} } \\
& \cdot\left[\theta\left(\rho\left(\widehat{S} r^{*}, r^{*}\right)+\rho\left(r^{*}, \widehat{S} r^{*}\right)\right)\right]^{d} \\
\leq & {\left[\theta\left(\rho\left(\widehat{S} r^{*}, r^{*}\right)\right)\right]^{a+2 d}<\theta\left(\rho\left(\widehat{S}\left(r^{*}\right), r^{*}\right)\right), }
\end{aligned}
$$

a contradiction. Thus, $\rho\left(\widehat{S}\left(r^{*}\right), r^{*}\right)=0$, and so $\widehat{S}\left(r^{*}\right)=r^{*}$. Again, since $\bar{T}$ is continuous, $\lim _{n \longrightarrow \infty} \breve{T}\left(g\left(r_{2 n+1}\right)\right)=\breve{T}\left(r^{*}\right)=\lim _{n \longrightarrow \infty} \breve{T}\left(\breve{T}\left(r_{2 n+1}\right)\right)$.

Since $(g, \breve{T})$ is a compatible pair,

$$
\lim _{n \longrightarrow \infty} \rho\left(g\left(\breve{T}\left(r_{2 n+1}\right)\right), \breve{T}\left(g\left(r_{2 n+1}\right)\right)\right)=0 .
$$

From Lemma 1, we have

$$
\lim _{n \longrightarrow \infty} g\left(\breve{T}\left(r_{2 n+1}\right)\right)=\breve{T}\left(r^{*}\right) .
$$

Put $r_{1}=r_{2 n}$ and $r_{2}=\breve{T}\left(r_{2 n+1}\right)$ in (89) and if $\rho\left(r^{*}, \breve{T}\left(r^{*}\right)\right)>0$, we obtain

$$
\frac{1}{2} \rho\left(f\left(r_{2 n}\right), \widehat{S}\left(r_{2 n}\right)\right)<\rho\left(\widehat{S}\left(r_{2 n}\right), \breve{T}\left(\breve{T}\left(r_{2 n+1}\right)\right)\right) .
$$

Therefore,

$$
\Upsilon\left(\begin{array}{c}
\rho\left(f\left(r_{2 n}\right), \widehat{S}\left(r_{2 n}\right)\right), \\
\rho\left(\widehat{S}\left(r_{2 n}\right), \breve{T}\left(\breve{T}\left(r_{2 n+1}\right)\right)\right)
\end{array}\right)<0,
$$

and from (89), one obtains

$$
\begin{aligned}
\theta\left(\rho\left(f\left(r_{2 n}\right), g\left(\breve{T}\left(r_{2 n+1}\right)\right)\right)\right) \leq & {\left[\theta\left(\rho\left(\widehat{S}\left(r_{2 n}\right), \breve{T}\left(\breve{T}\left(r_{2 n+1}\right)\right)\right)\right)\right]^{a} \cdot\left[\theta\left(\rho\left(f\left(r_{2 n}\right), \widehat{S}\left(r_{2 n}\right)\right)\right)\right]^{b} } \\
& \cdot\left[\theta\left(\rho\left(g\left(\breve{T}\left(r_{2 n+1}\right)\right), \breve{T}\left(\breve{T}\left(r_{2 n+1}\right)\right)\right)\right)\right]^{c} \\
\cdot & {\left[\theta\left(\rho\left(f\left(r_{2 n}\right), \breve{T}\left(\breve{T}\left(r_{2 n+1}\right)\right)\right)+\rho\left(g\left(\breve{T}\left(r_{2 n+1}\right)\right), \widehat{S}\left(r_{2 n}\right)\right)\right)\right]^{d} . }
\end{aligned}
$$

Passing to the upper limit in (128), we obtain

$$
\begin{aligned}
\theta\left(\rho\left(r^{*}, \breve{T}\left(r^{*}\right)\right)\right) & \leq\left[\theta\left(\rho\left(r^{*}, \breve{T}\left(r^{*}\right)\right)\right)\right]^{a+2 d} \\
& <\theta\left(\rho\left(r^{*}, \breve{T}\left(r^{*}\right)\right)\right)
\end{aligned}
$$

a contradiction. Thus, $\rho\left(r^{*}, \breve{T}\left(r^{*}\right)\right)=0$ and hence $r^{*}=T\left(r^{*}\right)$. Suppose that $\rho\left(f\left(r^{*}\right), r^{*}\right)>0$, we obtain

$$
\frac{1}{2} \rho\left(\widehat{S}\left(r^{*}\right), \breve{T}\left(r_{2 n+1}\right)\right)<\rho\left(f\left(r^{*}\right), \widehat{S}\left(r^{*}\right)\right), \quad \text { for all } n \in \mathbb{N},
$$

and so

$$
\Upsilon\left(\begin{array}{c}
\rho\left(f\left(r^{*}\right), \widehat{S}\left(r^{*}\right)\right), \\
\rho\left(\widehat{S}\left(r^{*}\right), \breve{T}\left(r_{2 n+1}\right)\right)
\end{array}\right)<0 .
$$

By (89), we obtain

$$
\begin{aligned}
\theta\left(\rho\left(f\left(r^{*}\right), g\left(r_{2 n+1}\right)\right)\right) \leq & {\left[\theta\left(\rho\left(\widehat{S}\left(r^{*}\right), \breve{T}\left(r_{2 n+1}\right)\right)\right)\right]^{a} \cdot\left[\theta\left(\rho\left(f\left(r^{*}\right), \widehat{S}\left(r^{*}\right)\right)\right)\right]^{b} } \\
& \cdot\left[\theta\left(\rho\left(g\left(r_{2 n+1}\right), \breve{T}\left(r_{2 n+1}\right)\right)\right)\right]^{c} \cdot\left[\theta\left(\rho\left(f\left(r^{*}\right), \breve{T}\left(r_{2 n+1}\right)\right)+\rho\left(g\left(r_{2 n+1}\right), \widehat{S}\left(r^{*}\right)\right)\right)\right]^{d} .
\end{aligned}
$$

Taking the upper limit in (132) and as $\theta\left(\rho\left(f\left(r^{*}\right), r^{*}\right)\right) \leq\left[\theta\left(\rho\left(f\left(r^{*}\right), r^{*}\right)\right)\right]^{b+d}<\theta\left(\rho\left(f\left(r^{*}\right), r^{*}\right)\right)$, 
a contradiction. Thus, $\rho\left(f\left(r^{*}\right), r^{*}\right)=0$ and hence $r^{*}=f\left(r^{*}\right)$. Finally, suppose $\rho\left(r^{*}, g\left(r^{*}\right)\right)>0$ and as $r^{*}=T\left(r^{*}\right)=\widehat{S}\left(r^{*}\right)=f\left(r^{*}\right)$, we have

$$
\Upsilon\left(0, \rho\left(r^{*}, g\left(r^{*}\right)\right)\right)<0 .
$$

From (89), we deduce

$$
\theta\left(\rho\left(r^{*}, g\left(r^{*}\right)\right)\right)=\theta\left(\rho\left(f\left(r^{*}\right), g\left(r^{*}\right)\right)\right)<\theta\left(\rho\left(r^{*}, g\left(r^{*}\right)\right)\right) \text {, }
$$

a contradiction. Thus, $\rho\left(r^{*}, g\left(r^{*}\right)\right)=0$ and $r^{*}=g\left(r^{*}\right)$. Therefore, $r^{*}$ is a common of the four mappings $\widehat{S}, g, f$, and $T$. Next, assume that $v^{*}$ is another common of the four mappings $\widehat{S}, g, f$, and $\widetilde{T}$ such that $r^{*} \neq v^{*}$, one obtains

$$
\Upsilon\left(0, \rho\left(\widehat{S}\left(r^{*}\right), \breve{T}\left(v^{*}\right)\right)\right)<0 .
$$

Then, by (89),

$$
\begin{aligned}
& \theta\left(\rho\left(r^{*}, v^{*}\right)\right)=\theta\left(\rho\left(\widehat{S}\left(r^{*}\right), \breve{T}\left(v^{*}\right)\right)\right) \leq\left[\theta\left(\rho\left(\widehat{S}\left(r^{*}\right), \breve{T}\left(v^{*}\right)\right)\right)\right]^{a} \cdot\left[\theta\left(\rho\left(f\left(r^{*}\right), \widehat{S}\left(r^{*}\right)\right)\right)\right]^{b} \\
& \cdot\left[\theta\left(\rho\left(g\left(v^{*}\right), \breve{T}\left(v^{*}\right)\right)\right)\right]^{c} \cdot\left[\theta\left(\rho\left(f\left(r^{*}\right), \breve{T}\left(v^{*}\right)\right)+\rho\left(g\left(v^{*}\right), \widehat{S}\left(r^{*}\right)\right)\right)\right]^{d} \cdot \\
& \theta\left(\rho\left(r^{*}, v^{*}\right)\right) \leq\left[\theta\left(\rho\left(r^{*}, v^{*}\right)\right)\right]^{a+2 d}<\theta\left(\rho\left(r^{*}, v^{*}\right)\right), \\
& \lim _{n \longrightarrow \infty} \rho\left(f \widehat{S}\left(r_{n}\right), \widehat{S} f\left(r_{n}\right)\right)=\lim _{n \longrightarrow \infty}\left|f \widehat{S}\left(r_{n}\right)-\widehat{S} f\left(r_{n}\right)\right| \\
& =|f(t)-\widehat{S}(t)|=|0-0|=0,
\end{aligned}
$$

a contradiction. Thus, $r^{*}=v^{*}$, that is, $r^{*}$ is the unique common fixed point of $\widehat{S}, g, f$, and $\widehat{T}$.

Example 6. Let $\chi=[0, \infty)$ and define the function $\rho: \chi \times$ $\chi \longrightarrow[0,+\infty)$ by $\rho(r, j)=|r-j|$. Clearly, $(\chi, \rho)$ is a complete metric space. Let $\theta(t)=e^{\sqrt{t}}$, and $\Upsilon(s, t)=(s / 2)-t$, then $\theta \in \Psi$ and $\Upsilon \in \Phi$. Define the mappings $\widehat{S}, g, f, \widehat{T}: \chi \longrightarrow \chi$ by

$$
\begin{aligned}
& \widehat{S}(r)=e^{7 r}-1, \\
& g(r)=\ln \left(1+\frac{r}{6}\right), \\
& f(r)=\ln \left(1+\frac{r}{6}\right), \\
& \breve{T}(r)=e^{6 r}-1 .
\end{aligned}
$$

Clearly, $f(\chi)=\breve{T}(\chi)=g(\chi)=\widehat{S}(\chi)$ if $\left\{r_{n}\right\}$ is a sequence in $\chi$ such that

$\lim _{n \longrightarrow \infty} f\left(r_{n}\right)=\lim _{n \longrightarrow \infty} \widehat{S}\left(r_{n}\right)=t, \quad$ for some $t \in \chi$.

Then,

$$
\lim _{n \longrightarrow \infty}\left|f\left(r_{n}\right)-t\right|=\lim _{n \longrightarrow \infty}\left|\widehat{S}\left(r_{n}\right)-t\right|=0,
$$

and equivalently,

$$
\lim _{n \longrightarrow \infty}\left|\ln \left(1+\frac{r_{n}}{6}\right)-t\right|=\lim _{n \longrightarrow \infty}\left|e^{7 r_{n}}-1-t\right|=0 .
$$

Thus,

$$
\lim _{n \longrightarrow \infty}\left|r_{n}-\left(6 e^{t}-6\right)\right|=\lim _{n \longrightarrow \infty}\left|r_{n}-\frac{\ln (1+t)}{7}\right|=0 .
$$

It gives that $6 e^{t}-6=(\ln (1+t) / 7)$ (by uniqueness of limit); hence, $t=0$. Using continuity of $f$ and $\widehat{S}$, one obtains for $t=0 \in \chi$. Hence, the pair $(f, \widehat{S})$ is compatible. Similarly, the pair $(g, T)$ is compatible. Next, for all $r, j \in \chi$ with

$$
\Upsilon(\rho(f r, \widehat{S} r), \rho(\widehat{S} r, \breve{T} j))<0 .
$$

So, for $a=b=c=0.1$ and $d=0.3$, we have

$$
\begin{aligned}
\theta(\rho(f r, g j)) \leq & {[\theta(\rho(\widehat{S} r, \breve{T} j))]^{a} \cdot[\theta(\rho(f r, \widehat{S} r))]^{b} } \\
\cdot & {[\theta(\rho(g j, \breve{T} j))]^{c} } \\
\cdot & {[\theta(\rho(f r, \breve{T} j)+\rho(g j, \widehat{S} r))]^{d} . }
\end{aligned}
$$

Hence, all hypotheses of Theorem 3 are satisfied. Thus, $f, g, \widehat{S}$, and $\widehat{T}$ have a unique common fixed point.

\section{An Application}

We apply the result given by Theorem 2 to study the existence of a solution for a system of nonlinear fractional differential equations. Let $\chi=C([0,1], \mathbb{R})$ be the space of all continuous functions on $[0,1]$. The metric on $\chi$ is given by

$$
\rho(r, j)=\|r-j\|_{\infty}=\max _{t \in[0,1]}|r(t)-j(t)|, \quad r, j \in \chi .
$$

Then, $\chi=C([0,1], \mathbb{R})$ is complete metric space.

Consider the following system of fractional differential equations:

$$
\left\{{ }^{C} D^{\alpha} r(t)=K_{1}(t, \widehat{S}(r(t))),{ }^{C} D^{\alpha} j(t)=K_{2}(t, \breve{T}(j(t))),\right.
$$

with boundary conditions

$$
\left\{\begin{array}{l}
r(0)=0, \operatorname{Ir}(1)=r^{\prime}(0), \\
j(0)=0, \operatorname{Qj}(1)=j^{\prime}(0) .
\end{array}\right.
$$


Note that ${ }^{C} D^{\alpha}$ denotes the Caputo fractional derivative of order $\alpha$, defined by

$$
\left\{{ }^{C} D^{\alpha} K_{1}(t)=\frac{1}{\Gamma(n-\alpha)} \int_{0}^{t}\left((t-s)^{n-\alpha-1} K_{1}^{n}(s)\right) \mathrm{d} s,{ }^{C} D^{\alpha} K_{2}(t)=\frac{1}{\Gamma(n-\beta)} \int_{0}^{t}\left((t-s)^{n-\alpha-1} K_{2}^{n}(s)\right) \mathrm{d} s\right.
$$

where we consider

$$
\begin{aligned}
n-1 & <\alpha, \\
\alpha & <1, \\
n & =[\alpha]+1,
\end{aligned}
$$

and $I^{\alpha} K_{1}$ and $I^{\alpha} K_{2}$ denote the Riemann-Liouville fractional integral of order $\alpha$ of continuous functions $K_{1}$ and $K_{2}$, given by

$$
\left\{\begin{array}{l}
I^{\alpha} K_{1}(t)=\frac{1}{\Gamma(\alpha)} \int_{0}^{t}(t-s)^{\alpha-1} K_{1}(s) \mathrm{d} s, \quad \text { with } \alpha>0 \\
Q^{\alpha} K_{2}(t)=\frac{1}{\Gamma(\alpha)} \int_{0}^{t}(t-s)^{\alpha-1} K_{2}(s) \mathrm{d} s, \quad \text { with } \alpha>0 .
\end{array}\right.
$$
form:

System (148) can be written in the following integral

$$
\left\{\begin{array}{l}
r(t)=\frac{1}{\Gamma(\alpha)} \int_{0}^{t}(t-s)^{\alpha-1} K_{1}(s, \widehat{S}(r(s))) \mathrm{d} s+\frac{2 t}{\Gamma(\alpha)} \int_{0}^{1} \int_{0}^{s}(s-u)^{\alpha-1} K_{1}(u, \widehat{S}(r(u))) \mathrm{d} u \mathrm{~d} s, \\
j(t)=\frac{1}{\Gamma(\alpha)} \int_{0}^{t}(t-s)^{\alpha-1} K_{2}(s, \breve{T}(j(s))) \mathrm{d} s+\frac{2 t}{\Gamma(\alpha)} \int_{0}^{1} \int_{0}^{s}(s-u)^{\alpha-1} K_{2}(u, \breve{T}(j(u))) \mathrm{d} u \mathrm{~d} s .
\end{array}\right.
$$

Define the mappings $f, g: \chi \longrightarrow \chi$ by

$$
\begin{array}{r}
\left\{f(r(t))=\frac{1}{\Gamma(\alpha)} \int_{0}^{t}(t-s)^{\alpha-1} K_{1}(s, \hat{S}(r(s))) \mathrm{d} s \frac{2 t}{\Gamma(\alpha)} \int_{0}^{1} \int_{0}^{s}(s-u)^{\alpha-1} K_{1}(u, \hat{S}(r\{u)) \mathrm{d} u \mathrm{~d} s,\right. \\
g(j(t))=\frac{1}{\Gamma(\alpha)} \int_{0}^{t}(t-s)^{\alpha-1} K_{2}(s, \breve{T}(j(s))) \mathrm{d} s+\frac{2 t}{\Gamma(\alpha)} \int_{0}^{1} \int_{0}^{s}(s-u)^{\alpha-1} K_{2}(u, \breve{T}(j(u))) \mathrm{d} u \mathrm{~d} s .
\end{array}
$$

Theorem 4. Assume that the following conditions hold:

$$
\left|K_{1}(s, \widehat{S}(r(s)))-K_{2}(s, \breve{T}(j(s)))\right| \leq \frac{e^{-\tau} \Gamma(\alpha+1)}{4} A(r, j),
$$

(i) $K_{1}, K_{2}[0,1] \times \mathbb{R} \longrightarrow \mathbb{R}$ are continuous functions.

(ii) $K_{1}(s,),. K_{2}(s,):. \longrightarrow \mathbb{R}$ are increasing functions.

(iii) For all $r, j \in \chi$ with $f(r) \leq \bar{T}(j)$ and

where

$|f(r(s))-g(j(s))|>0$, we have

$$
A(r, j)=\max \left\{\begin{array}{c}
|\widehat{S}(r(s))-\breve{T}(j(s))|,|f(r(s))-\widehat{S}(r(s))|, \\
|g(j(s))-\breve{T}(j(s))|, \frac{|f(r(s))-\breve{T}(j(s))|+|g(j(s))-\widehat{S}(r(s))|}{2}, \\
\frac{(1+|f(r(s))-\widehat{S}(r(s))|)|g(j(s))-\breve{T}(j(s))|}{1+|\widehat{S}(r(s))-\breve{T}(j(s))|}
\end{array}\right\} .
$$


(iv) There exists $r_{0}, j_{0} \in C([0,1], \mathbb{R})$ such that, for all $t \in[0,1]$, we have

$$
\left\{\begin{array}{l}
r_{0}(t) \leq \frac{1}{\Gamma(\alpha)} \int_{0}^{t}(t-s)^{\alpha-1} K_{1}\left(s, \widehat{S}\left(r_{0}(s)\right)\right) \mathrm{d} s+\frac{2 t}{\Gamma(\alpha)} \int_{0}^{1} \int_{0}^{s}(s-u)^{\alpha-1} K_{1}\left(u, \widehat{S}\left(r_{0}(u)\right)\right) \mathrm{d} u \mathrm{~d} s, \\
j_{0}(t) \leq \frac{1}{\Gamma(\alpha)} \int_{0}^{t}(t-s)^{\alpha-1} K_{2}\left(s, \breve{T}\left(j_{0}(s)\right)\right) \mathrm{d} s+\frac{2 t}{\Gamma(\alpha)} \int_{0}^{1} \int_{0}^{s}(s-u)^{\alpha-1} K_{2}\left(u, \breve{T}\left(j_{0}(u)\right)\right) \mathrm{d} u \mathrm{~d} s .
\end{array}\right.
$$

(v) If there exists a sequence $\left\{r_{n}\right\}$ in $\chi$ such that

$$
\begin{aligned}
\lim _{n \longrightarrow \infty} \rho\left(f\left(\widehat{S}\left(r_{n}\right)\right), \widehat{S}\left(f\left(r_{n}\right)\right)\right) & =0, \\
\lim _{n \longrightarrow \infty} \rho\left(g\left(\breve{T}\left(r_{n}\right)\right), \breve{T}\left(g\left(r_{n}\right)\right)\right) & =0,
\end{aligned}
$$

whenever

$$
\begin{aligned}
& \lim _{n \longrightarrow \infty} f\left(r_{n}\right)=\lim _{n \longrightarrow \infty} \widehat{S}\left(r_{n}\right)=t, \\
& \lim _{n \longrightarrow \infty} g\left(r_{n}\right)=\lim _{n \longrightarrow \infty} \breve{T}\left(r_{n}\right)=t,
\end{aligned}
$$

for some $t \in \chi$.

Then, system (148) has a solution.

Proof. Following assumptions (iii) and (iv), we have

$$
\begin{aligned}
& |f(r(t))-g(j(t))| \\
& =\left|\begin{array}{c}
\frac{1}{\Gamma(\alpha)} \int_{0}^{t}(t-s)^{\alpha-1} K_{1}(s, \widehat{S}(r(s))) \mathrm{d} s \\
-\frac{1}{\Gamma(\alpha)} \int_{0}^{t}(t-s)^{\alpha-1} K_{2}(s, \check{T}(j(s))) \mathrm{d} s \\
+\frac{2 t}{\Gamma(\alpha)} \int_{0}^{1} \int_{0}^{s}(s-u)^{\alpha-1} K_{1}(u, \widehat{S}(r(u))) \mathrm{d} u \mathrm{~d} s \\
-\frac{2 t}{\Gamma(\alpha)} \int_{0}^{1} \int_{0}^{s}(s-u)^{\alpha-1} K_{2}(u, \check{T}(j(u))) \mathrm{d} u \mathrm{~d} s
\end{array}\right| \\
& \leq \frac{1}{\Gamma(\alpha)} \int_{0}^{t}(t-s)^{\alpha-1}\left|K_{1}(s, \widehat{S}(r(s)))-K_{2}(s, \check{T}(j(s)))\right| \mathrm{d} s \\
& +\frac{2}{\Gamma(\alpha)} \int_{0}^{1} \int_{0}^{s}(s-u)^{\alpha-1}\left|K_{1}(s, \widehat{S}(r(s)))-K_{2}(u, \check{T}(j(u)))\right| \mathrm{d} u \mathrm{~d} s \\
& \leq \frac{1}{\Gamma(\alpha)} \frac{e^{-\tau} \Gamma(\alpha+1)}{4} \cdot \int_{0}^{t}(t-s)^{\alpha-1} A(r, j) \mathrm{d} s \\
& \quad+\frac{2}{\Gamma(\alpha)} \frac{e^{-\tau} \Gamma(\alpha+1)}{4} \cdot \int_{0}^{1} \int_{0}^{s}(s-u)^{\alpha-1} A(r, j) \mathrm{d} u \mathrm{~d} s \\
& \leq \frac{1}{\Gamma(\alpha)} \frac{e^{-\tau} \Gamma(\alpha+1)}{4} \cdot A(r, j) \cdot \int_{0}^{t}(t-s)^{\alpha-1} \mathrm{~d} s
\end{aligned}
$$




$$
\begin{aligned}
& +\frac{2}{\Gamma(\alpha)} \frac{e^{-\tau} \Gamma(\alpha+1)}{4} \cdot A(r, j) \cdot \int_{0}^{1} \int_{0}^{s}(s-u)^{\alpha-1} \mathrm{~d} u \mathrm{~d} s \\
\leq & \left(\frac{e^{-\tau} \Gamma(\alpha) \cdot \Gamma(\alpha+1)}{4 \Gamma(\alpha) \cdot \Gamma(\alpha+1)}\right) \cdot A(r, j)+2 e^{-\tau} B(\alpha+1,1) \frac{\Gamma(\alpha) \cdot \Gamma(\alpha+1)}{4 \Gamma(\alpha) \cdot \Gamma(\alpha+1)} \cdot A(r, j) \\
\leq & \frac{e^{-\tau}}{4} A(r, j)+\frac{e^{-\tau}}{2} A(r, j),
\end{aligned}
$$

where $B$ is the beta function. From the above inequality, we obtain that

$$
\rho(f(r), g(j)) \leq \frac{3}{4} e^{-\tau} A(r, j) .
$$

It implies that

$$
\begin{aligned}
e^{\sqrt{\rho(f(r), g(j))}} & \leq e^{\sqrt{(3 / 4) e^{-\tau} A(r, j)}}=e^{\sqrt{(3 / 4) e^{-\tau}} \sqrt{A(r, j)}} \\
& =\left[e^{\sqrt{A(r, j)}}\right]^{\sqrt{(3 / 4) e^{-\tau}}}=\left[e^{\sqrt{A(r, j)}}\right]^{E(G(r, j))},
\end{aligned}
$$

where $\quad E\left(s_{1}, s_{2}, s_{3}, s_{4}\right)=\sqrt{3 / 4 e^{\tau}}<1, G(r, j)=$ $\{\rho(f r, \widehat{S} r), \rho(g j, \breve{T} j), \rho(f r, \breve{T} j), \rho(g j, \widehat{S} r)\}$ and $\theta(t)=e^{\sqrt{t}}$. Since this inequality holds for all $r, j \in \chi$ with $f(r) \leq \breve{T}(j)$, so it is true for any $\Upsilon \in \Phi$,

$$
\Upsilon(\rho(f r, \widehat{S} r), \rho(\widehat{S} r, \breve{T} j))<0 .
$$

Hence, we have

$$
\theta(\rho(f(r), g(j))) \leq[\theta(A(r, j))]^{E(G(r, j))} .
$$

Thus, $f, g, \widehat{S}$, and $\breve{T}$ are generalized Suzuki-type $(\theta, E)$-rational contraction mappings. Therefore, all hypotheses of Theorem 2 are satisfied. Hence, $f, g, \widehat{S}$, and $T$ have a common fixed point, that is, system (148) has at least one solution.

\section{Data Availability}

No data were used to support this study.

\section{Conflicts of Interest}

The authors declare that they have no conflicts of interest.

\section{Authors' Contributions}

All authors read and approved the final manuscript.

\section{Acknowledgments}

Vahid Parvaneh dedicates this article to the first teacher, Mr. Allah Karam Parsaei, of the village where he was born, for his hard work and efforts in education during the years 19751977.

\section{References}

[1] S. Banach, "Sur les opérations dans les ensembles abstraits et leur application aux équations intégrales," Fundamenta Mathematicae, vol. 3, pp. 133-181, 1922.

[2] M. Abbas, H. Iqbal, and A. Petrusel, "Fixed points for multivalued Suzuki type $(\theta, R)$-contraction mapping with applications," Journal of Function Spaces, vol. 2019, p. 13, Article ID 9565804, 2019.

[3] H. Aydi, E. Karapinar, and W. Shatanawi, "Coupled fixed point results for $(\psi, \varphi)$-weakly contractive condition in ordered partial metric spaces," Computers and Mathematics with Applications, vol. 62, pp. 4449-4460, 2011.

[4] H. Aydi, E. Karapinar, and A. Roldán López de Hierro, " $\omega$-interpolative cirić-reich-rus-type contractions," Mathematics, vol. 7, no. 1, p. 57, 2019.

[5] M. Nazam, H. Aydi, and M. S. Noorani, "Existence of fixed points of four maps for a new generalized F-contraction and an application," Journal of Function Spaces, vol. 2019, Article ID 5980312, 8 pages, 2019.

[6] M. Nazam, M. Arshad, and M. Postolache, "Coincidence and common fixed point theorems for four mappings satisfying $(\alpha \mathrm{s}, \mathrm{F})$-contraction $\left.\alpha^{\mathrm{s}} \mathrm{F}\right)$-contraction," Nonlinear Analysis: Modelling and Control, vol. 23, no. 5, pp. 664-690, 2018.

[7] M. Nazam, M. Arshad, and M. Abbas, "Existence of common fixed points of improved F-contraction on partial metric spaces," Applied General Topology, vol. 18, no. 2, pp. 277-287, 2017.

[8] H. Aydi, C.-M. Chen, and E. Karapınar, "Interpolative cirićreich-rus type contractions via the branciari distance," Mathematics, vol. 7, no. 1, p. 84, 2019.

[9] E. Karapinar, R. Agarwal, and H. Aydi, "Interpolative reichrus-cirić type contractions on partial metric spaces," Mathematics, vol. 6, no. 11, p. 256, 2018.

[10] E. Karapinar, O. Alqahtani, and H. Aydi, "On interpolative Hardy-Rogers type contractions," Symmetry, vol. 11, no. 1, p. 8, 2019.

[11] T. Rasham and A. Shoaib, "Common fixed point results for two families of multivalued A-dominated contractive mappings on closed ball with applications," Open Mathematics, vol. 17, no. 1, pp. 1350-1360, 2019.

[12] T. Rasham, A. Shoaib, G. Marino, B. A. S. Alamri, and M. Arshad, "Sufficient conditions to solve two systems of integral equations via fixed point results," Journal of Inequalities and Applications, vol. 2019, Article ID 182, 2019.

[13] P. Patle, D. Patel, H. Aydi, and S. Radenović, "ON H ${ }^{+}$type multivalued contractions and applications in symmetric and probabilistic spaces $\mathrm{H}^{+}$-type multivalued contractions and applications in symmetric and probabilistic spaces," Mathematics, vol. 7, no. 2, p. 144, 2019.

[14] N. Alamgir, Q. Kiran, H. Isik, and H. Aydi, "Fixed point results via a Hausdorff controlled type metric," Advances in Difference Equations, vol. 2020, Article ID 24, 2020. 
[15] E. Ameer, H. Aydi, M. Arshad, and M. De la Sen, "Hybrid Ćirić type graphic $\Upsilon, \Lambda$-contraction mappings with applications to electric circuit and fractional differential equations $(\Upsilon$, $\Lambda)$-contraction mappings with applications to electric circuit and fractional differential equations," Symmetry, vol. 12, no. 3 , p. 467, 2020.

[16] T. Rasham, A. Shoaib, N. Hussain, B. A. S. Alamri, and M. Arshad, "Multivalued fixed point results in dislocated b-metric spaces with application to the system of nonlinear integral equations," Symmetry, vol. 11, no. 1, p. 40, 2019.

[17] E. Ameer, H. Aydi, M. Arshad, H. Alsamir, and M. Noorani, "Hybrid multivalued type contraction mappings in $\alpha_{K^{-}}$ complete partial $b$-metric spaces and applications," Symmetry, vol. 11 , no. 1, p. $86,2019$.

[18] H. Aydi, H. Lakzian, Z. D. Mitrović, and S. Radenović, "Best proximity points of MT-cyclic contractions with property UC," Numerical Functional Analysis and Optimization, vol. 41, no. 7, pp. 871-882, 2020.

[19] E. Karapinar, S. Czerwik, and H. Aydi, "( $\alpha, \psi)$-Meir-Keeler contraction mappings in generalized b-metric spaces," Journal of Function Spaces, vol. 2018, Article ID 3264620, 4 pages, 2018.

[20] T. Suzuki, "A generalized Banach contraction principle which characterizes metric completeness," Proceedings of the American Mathematical Society, vol. 136, pp. 1861-1870, 2007.

[21] H. Piri and P. Kumam, "Some fixed point theorems concerning F-contraction in complete metric spaces," Fixed Point Theory and Applications, vol. 2014, Article ID 210, 2014.

[22] H. Piri and P. Kumam, "Fixed point theorems for generalized F-Suzuki-contraction mappings in complete $b$-metric spaces," Fixed Point Theory and Applications, vol. 2016, Article ID 90, 2016.

[23] H. Aydi, M. A. Barakat, Z. D. Mitrovic, and V. Sesum-Cavi, "A Suzuki-type multivalued contraction on weak partial metric spaces and applications," Journal of Inequalities and Applications, vol. 2018, Article ID 270, 2018.

[24] M. Jleli and B. Samet, "A new generalization of the Banach contraction principle," Journal of Inequalities and Applications, vol. 2014, Article ID 38, 2014

[25] M. Jleli, E. Karapınar, and B. Samet, "Further generalizations of the Banach contraction principle," Journal of Inequalities and Applications, vol. 2014, no. 1, Article ID 439, 2014.

[26] Z. Li and S. Jiang, "Fixed point theorems of JS-quasi-contractions," Fixed Point Theory and Applications, vol. 2016, Article ID 40, 2016.

[27] I. Altun, A. Al, M. Jleli, A. Lashine, and B. Samet, “A Fixed point theorem for JS-contraction type mappings with applications to polynomial approximations," Filomat, vol. 31, no. 15, pp. 4969-4978, 2017.

[28] J. Ahmed, A. E. Al-Mazrooei, Y. J. Cho, and Y. O. Yang, "Fixed point results for generalized $\Theta$-contractions," Journal of Nonlinear Sciences and Applications, vol. 10, pp. 2350-2358, 2017.

[29] N. Hussain, V. Parvaneh, B. Samet, and C. Vetro, "Some fixed point theorems for generalized contractive mappings in complete metric spaces," Fixed Point Theory and Applications, vol. 2015, Article ID 185, 2015.

[30] N. Hussain, A. E. Al-Mazrooei, and J. Ahmad, "Fixed point results for generalized $(\alpha-\eta)-\Theta$ contractions with applications $\alpha, \eta)-\theta$-contractions with applications," The Journal of Nonlinear Sciences and Applications, vol. 10, no. 8, pp. 4197-4208, 2017.

[31] V. Parvaneh, F. Golkarmanesh, N. Hussain, and P. Salimi, "New fixed point theorems for $\alpha-\mathrm{H} \theta$ contractions in ordered metric spaces," Journal of Fixed Point Theory and Applications, vol. 18, no. 4, pp. 905-925, 2016.

[32] H. A. Alolaiyan, B. Ali, and M. Abbas, "Fixed point results of Edelstein-Suzuki type multivalued mappings on $b$-metric spaces with applications b-metric spaces with applications," The Journal of Nonlinear Sciences and Applications, vol. 10, no. 3, pp. 1201-1214, 2017.

[33] X. Liu, S. Chang, Y. Xiao, and L. C. Zhao, "Existence of fixed points for $\Theta$-type contraction and $\Theta$-type Suzuki contraction in complete metric spaces," Fixed Point Theory and Applications, vol. 2016, no. 1, 2016.

[34] V. M. Sehgal, "On fixed and periodic points for a class of mappings," Journal of the London Mathematical Society, vol. s2-5, no. 3, pp. 571-576, 1972. 